\title{
Violation of Hund's Rule in Molecules: Predicting the Excited-State Energy Inversion by TD-DFT with Double-Hybrid Methods
}

Accepted Manuscript: This article has been accepted for publication and undergone full peer review but has not been through the copyediting, typesetting, pagination, and proofreading process, which may lead to differences between this version and the Version of Record.

Cite as: J. Chem. Phys. (in press) (2021); https://doi.org/10.1063/5.0076545

Submitted: 26 October 2021 - Accepted: 02 December 2021 • Accepted Manuscript Online: 02 December 2021

(iD) Juan Carlos Sancho-Garcia, (D) Eric Bremond, Gaetano Ricci, et al.
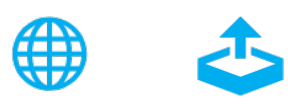

\section{ARTICLES YOU MAY BE INTERESTED IN}

The Devil's Triangle of Kohn-Sham density functional theory and excited states

The Journal of Chemical Physics 154, 074106 (2021); https://doi.org/10.1063/5.0035446

Reliable transition properties from excited-state mean-field calculations

The Journal of Chemical Physics 154, 124106 (2021); https://doi.org/10.1063/5.0041233

Revealing the nature of electron correlation in transition metal complexes with symmetry breaking and chemical intuition

The Journal of Chemical Physics 154, 194109 (2021); https://doi.org/10.1063/5.0047386

\section{Challenge us.}

What are your needs for periodic signal detection?
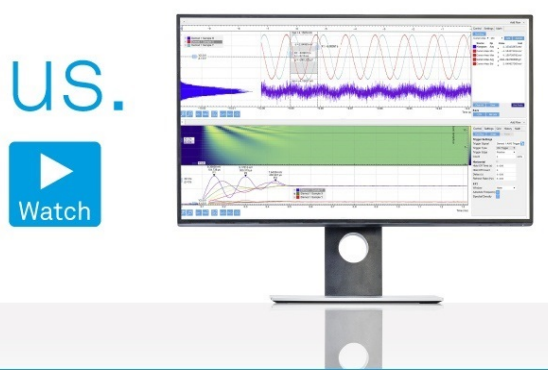

Zurich Instruments 


\section{Violation of Hund's Rule in Molecules: Predicting the Excited-State Energy Inversion by TD-DFT with Double-Hybrid Methods}

J. C. Sancho-García ${ }^{*}$, E. Brémond ${ }^{b}$, G. Ricci ${ }^{c}$, A.J. Pérez-Jiménez ${ }^{a}$, Y. Olivier ${ }^{c}$, and C. Adamo ${ }^{d, e}$

${ }^{a}$ Department of Physical Chemistry,

University of Alicante,

E-03080 Alicante, Spain

${ }^{b}$ Université de Paris, ITODYS, CNRS,

F-75006 Paris, France

${ }^{c}$ Unité de Chimie Physique Théorique et Structurale

\& Laboratoire de Physique du Solid,

Namur Institute of Structured Matter,

Université de Namur,

B-5000 Namur, Belgium

${ }^{d}$ Chimie ParisTech, PSL Research University, CNRS,

Institute of Chemistry for Life and Health Sciences (i-CLeHS), FRE 2027, F-75005 Paris, France

${ }^{e}$ Institut Universitaire de France, 103 Boulevard Saint Michel, F-75005 Paris, France

November 27, 2021

*E-mail: jc.sancho@ua.es 


\begin{abstract}
The energy difference $\left(\Delta E_{S T}\right)$ between the lowest singlet $\left(S_{1}\right)$ and triplet $\left(T_{1}\right)$ excited state of a set of azaphenalene compounds, which is theoretically and experimentally known to violate Hund's rule giving rise to the inversion of the order of those states, is calculated here with a family of double-hybrid density functionals. That excited-state inversion is known to be very challenging to reproduce for TD-DFT employing common functionals, e.g. hybrid or range-separated expressions, but not for wavefunction methods due to the inclusion of higher-thansingle excitations. Therefore, we explore here if the last developed family of density functional expressions (i.e., double-hybrid models) is able to provide not only the right excited-state energy order but also accurate $\Delta E_{S T}$ values, thanks to the approximate inclusion of double excitations within these models. We herein employ standard double-hybrid (B2-PLYP, PBE-QIDH, and PBE0-2), range-separated ( $\omega$ B2-PLYP and RSX-QIDH), spin-scaled (SCS/SOS-B2PLYP21, SCS-PBE-QIDH, and SOS-PBE-QIDH), and range-separated spin-scaled (SCS/SOS- $\omega$ B2-PLYP, SCS-RSX-QIDH, and SOS-RSX-QIDH) expressions, to systematically assess the influence of the ingredients entering into the formulation while concomitantly providing insights for their accuracy.
\end{abstract}




\section{Introduction}

In the vast majority of organic chromophores, the lowest singlet excited state $\left(S_{1}\right)$ lies energetically above the lowest triplet excited state $\left(T_{1}\right)$ due to the fact that the exchange energy is known to be positive, and so it is the corresponding singlet-triplet energy difference or $\Delta E_{S T}$. In a first-order approximation (i.e., a single-particle excitation framework) the energy difference between both excited states equals $2 K_{i a}$, which is twice the exchange energy $\left(K_{i a}\right)$ depending on the corresponding occupied $\left(\phi_{i}\right)$ and virtual $\left(\phi_{a}\right)$ orbitals involved in the spin-dependent formation of the excited-states. If $\Delta E_{0 S}\left(\Delta E_{0 T}\right)$ corresponds to the energy difference between the ground- $\left(S_{0}\right)$ and the $S_{1}\left(T_{1}\right)$ excited-state, then $\Delta E_{0 S} \approx \Delta E_{0 T}+2 K_{i a}$. The last term is known to be of the order of $0.5-1.0 \mathrm{eV}$ for conjugated polymers, ${ }^{1-3}$ but smaller for finite-size molecules as polycyclic conjugated hydrocarbons. That Hund's rule for molecules is also expected to hold ${ }^{4}$ within Density Functional Theory (DFT). However, if correlation effects are taken into account, the zeroth order approximation is not completely valid and that singlet-triplet energy difference can decrease ${ }^{5,6}$ and thus affects many photophysical processes. ${ }^{7}$ Therefore, the degree of excited-states correlation effects, and how theoretical methods are able to deal with it, is a topic of utmost importance for further materials design.

Actually, and generally speaking, if the singlet-triplet energy difference $\Delta E_{S T}=\Delta E_{0 S}-\Delta E_{0 T}$ becomes low enough (e.g. in the order of 100-200 $\mathrm{meV}$ ) it could be expected to foster a Reverse Intersystem Crossing Process (RISC), thus appreciably populating the $S_{1}$ state above the spin statistics limit (a 1:3 ratio for the singlet:triplet exciton formation). This mechanism has already found promising applications in last years for light-emitting devices ${ }^{8-14}$ since it could recover a significant portion of the dark (triplet) excitons naturally formed upon electroluminiscence. This recovery could ideally enhance the 
quantum yield, contributing to more efficient Organic Light-Emitting Diodes (OLEDs) through a mechanism known as Thermally Activated Delayed Fluorescence (TADF) process. The conditions under which a chemical compound could behave as a TADF emitter are being thoroughly investigated theoretically and experimentally. ${ }^{15-19}$ The first attempts relied on the smart combination of chemical fragments acting as a donor or as an acceptor, giving rise upon excitation to intra-molecular charge-transfer excitations with small exchange energies ${ }^{20}$ and thus correspondingly displaying low $\Delta E_{S T}$ values. A step further was later achieved after the discovery of compounds holding almost isoenergetic $S_{1}$ and $T_{1}$ excited-state energies, promoting very small (of the order of $100 \mathrm{meV}$, but still positive) $\Delta E_{S T}$ values. ${ }^{21-23}$ Theoretical investigations also helped to isolate the reasons for such small values, which it was not only due to small exchange energies but mostly to correlation effects further contributing to the decrease of $\Delta E_{S T} \cdot{ }^{24,25}$

The next step which could be envisioned would be the existence of compounds displaying negative $\Delta E_{S T}$ values after the inversion of the $S_{1}$ and $T_{1}$ excited states, which could thus foster the barrierless downconversion of triplet to singlet excitons to achieve the promise of a $100 \%$ of quantum efficiency. This is not a so rare effect as it could be initially thought, since some old studies by Leupin et al. in the 80s already found solid evidences of that energy inversion for a set of chemical systems known as azaphenalenes. ${ }^{26,27} \mathrm{~A}$ recent review ${ }^{28}$ by Audebert $e$ t al. reports on the synthetic versatility and different achievements realized from that kind of molecules, be monomeric or not, which includes many fields of applications (photocatalysts, light-emitting diodes, covalent organic frameworks, liquid crystals, etc.) The advances in the synthesis of those compounds, together with the possibility of that excited-state energy inversion, clearly opens a wide area of research still not fully explored. ${ }^{29}$ If that excited-state energy inversion is possible and favourable, it must be theoretically explored by correlated methods beyond the single-excitation pic- 
ture $^{30,31}$ which is however not possible within the linear-response (adiabatic) Time-Dependent Density Functional Theory (TD-DFT) method as recently shown. ${ }^{32}$

Unsurprisingly, wavefunction methods for excited states can predict that excited-state energy inversion if at least double excitations are allowed into the excited-state wavefunction, ${ }^{33-35}$ although at a normally more demanding cost than TD-DFT. A way to reconcile these two extremes; i.e., the need to introduce double excitations, together with the vast implementation of TD-DFT in the most used computational codes, could be the use of double-hybrid density functionals ${ }^{36-42}$ for which a double correction is added to the zeroth-order excitation energies. We will thus systematically explore here the accuracy of this family of methods, which are recognized to considerably improve the performance of the well-known hybrid functionals for many properties, ${ }^{43}$ for that set of azaphenalenes compounds known to display the excited-state energy inversion. We will also aim at contributing to better understand the performance of these expressions as a function of their composition, since many approaches and variants bloomed in the last years, and try to rationalize previous applications of double-hybrid density functionals to this challenging issue. ${ }^{33}$

\section{Justification of the choice of target systems}

We have started the selection of the target systems with the prototypical set of azaphenalene molecules largely tackled in previous studies, see Figure 1 , and for which a negative $\Delta E_{S T}$ value is robustly predicted and vastly reproduced by wavefunction methods from some pioneering works ${ }^{33-35}$ to more recent ones. ${ }^{32,44,45}$ The conclusions of the study by Ehrmaier et al. ${ }^{34}$ also found that subsitution of the heptazine core $-\mathrm{C}_{6} \mathrm{H}_{7} \mathrm{H}_{3}-$ with chlorine $-\mathrm{C}_{6} \mathrm{H}_{7} \mathrm{Cl}_{3}-$, cyano $-\mathrm{C}_{6} \mathrm{H}_{7}(\mathrm{CN})_{3^{-}}$, or p- 
methoxyphenylene $-\mathrm{C}_{6} \mathrm{~N}_{7}\left(\mathrm{p}-\mathrm{C}_{6} \mathrm{H}_{4} \mathrm{OCH}_{3}\right)_{3}-$ groups also preserved the negative $\Delta E_{S T}$ value. However, these compounds are known to display negligible, or very small in the best of the cases, transition dipole moments and associated oscillator strengths, which disqualify them for efficient light-emitting applications. On the other hand, the controlled chemical modification of these systems, by increasing the size of the triangular-shaped core and/or by introducing $\mathrm{N}$ or $\mathrm{B}$ atoms into specific positions of the structure, leads also to systems showing $\Delta E_{S T}<0$ values and non-negligible oscillator strengths, ${ }^{46}$ which indicates the vast possibilities still unexplored for these unique structures.

Motivated by all these previous findings, a massive yet robust screening ${ }^{47}$ of thousands of potential azaphenalene candidates was recently accomplished by Pollice et al. ${ }^{48}$ The systems were allowed to vary their topology (i.e., fusing aromatic rings to the triangular-shaped core), their $\mathrm{N}$ content (i.e., the number of C-substituted sites) and their peripherical groups to allow for the computationally smart data-driven design of dyes with negative $\Delta E_{S T}$ values together with reasonably non-negligible (and often large) oscillator strengths, normally found to be antagonistic properties. That pioneering study motivated us to complete our investigation with some of the most promising molecules presented in Figure 2 from the study of Pollice et al., still not-yet-synthesized but having sufficiently negative $\Delta E_{S T}$ values.

Finally, we have also included a real-world heptazine-based organic dye recently incorporated into an $\mathrm{OLED}^{49,50}$ (see its chemical structure in Figure 3). The device uses the 2,5,8-tris(4-fluoro-3-methylphenyl)-1,3,4,6,7,9,9bheptaazaphenalene (HAP-3MF) system: a dye based on the 2T-7N (or heptazine core) incorporating the fluorotoluene substituent to enhance the solubility and the electron accepting character of pristine heptazine. This device exhibits an exceptionally high quantum efficiency, which is explained by the 
large recovery of dark triplet excitons since prompt and delayed emissions are found nearly identical for an exciplex consisting of HAP-3MF (acceptor) and 1,3-bis(N-carbazolyl)benzene or $\mathrm{mCP}$ (donor). ${ }^{51}$ This system was also recently studied by Domcke and Sobolewski, finding a $\Delta E_{S T}<0$ by wavefunction methods ${ }^{52}$ and thus confirming the excited-state energy inversion.

\section{Theoretical methods and associated details}

\subsection{Generalities about double-hybrid density functionals}

To fully understand the performance of a Double-Hybrid (DH) density functional for excited states, which is the main goal of the present study, we recall here their (simplified) general expression:

$$
E_{x c}^{\mathrm{DH}}[\rho]=a_{x} E_{x}^{\mathrm{EXX}}\left[\phi_{i}\right]+\left(1-a_{x}\right) E_{x}[\rho]+a_{c} E_{c}^{\mathrm{PT} 2}\left[\phi_{i}, \phi_{a}\right]+\left(1-a_{c}\right) E_{c}[\rho],
$$

whose exchange contribution is a combination of EXact-eXchange (EXX) $E_{x}^{\mathrm{EXX}}$ and exchange functional $E_{x}[\rho], a_{x}$ being the coefficient of the former term, and the correlation contribution is also a combination of a second-order Perturbation Theory (PT2) term $E_{c}^{\mathrm{PT} 2}$ and the correlation functional $E_{c}[\rho]$, with $a_{c}$ being the weight given to the former term. Note that $\phi_{i}\left(\phi_{a}\right)$ are the occupied (unoccupied) orbitals self-consistently obtained from the underlying single-hybrid functional, which neglects the PT2 contribution: that is, $E_{x c}[\rho]=a_{x} E_{x}^{\operatorname{EXX}}\left[\phi_{i}\right]+\left(1-a_{x}\right) E_{x}[\rho]+\left(1-a_{c}\right) E_{c}[\rho]$. The latter hybrid expression would give an excitation energy $(\Omega)$ if applied with the linear-response formalism, as any other hybrid functional, from which one could better understand the key role played by the $a_{c}$ weight for excitation energies: the final excitation energies obtained from the corresponding double-hybrid expression $\left(\Omega^{\prime}\right)$ are given by

$$
\Omega^{\prime}=\Omega+a_{c} \Delta(\mathrm{D})
$$

with $\Delta(\mathrm{D})$ the corresponding correction for excited states $^{53}$ leading thus to the (D)-corrected excitation energy $\Omega^{\prime} \cdot{ }^{54,55}$ We would like to emphasize here 
that the values of $a_{x}$ and $a_{c}$ can be found following roughly two different strategies: after their fitting to some training dataset, and thus necessarily introducing some parameterization, ${ }^{56,57}$ or by imposing a set of known exact constraints and known theoretical limits, with the corresponding expressions being considered as minimally empirical models. ${ }^{58-60}$

Some other routes, such as the range-separation of the exchange term, are also deeply investigated to improve the previous expressions, be for groundor excited-states properties. This strategy is based on the splitting of the electron-repulsion operator $r_{i j}^{-1}$ using the error function:

$$
\frac{1}{r_{i j}}=\frac{1-\left[\alpha+\beta \operatorname{erf}\left(\omega r_{i j}\right)\right]}{r_{i j}}+\frac{\alpha+\beta \operatorname{erf}\left(\omega r_{i j}\right)}{r_{i j}}
$$

where $\alpha$ and $\alpha+\beta$ are coefficients used to weight the short- and long-range EXX, respectively, with the range-separation parameter $\omega$ governing the separation between the two regimes. This extension borrows the same argument than that previously one used for hybrid functionals; i.e., improving longrange electronic properties by gradually allowing the recovery of the correct asymptotic behaviour. ${ }^{61-63}$ The $\omega$ value can be again tuned for ground- or excited-state properties, ${ }^{64}$ or fitted from first-principle approaches ${ }^{65-67}$ again consistently with parameterized or minimally empirical models strategies, respectively. Despite being the most often used approach, there are also some recent examples for which the long-range correction is applied to both exchange and correlation terms. ${ }^{68}$ Additionally, we also mention specifically here the lrc-XYG3 functional, ${ }^{69}$ where range-separation applies to the correlation part with increasing PT2 contribution at the long range, to show the diversity of existing choices.

Finally, since the PT2 contribution could be seen as a too crude truncation of the perturbative series ${ }^{70}$ it is also common to scale differently the same- and opposite-spin energy contribution of that term, giving rise to Spin- 
Component-Scaled (SCS) double-hybrid models, be range-separated or not. This strategy has been recently pursued for both set of functionals, parameterized $^{71}$ or minimally empirical, ${ }^{72}$ mostly for ground-state properties ${ }^{73-76}$ unless some recent contributions for excited states too. ${ }^{77,78}$ Note that any spin-scaled method can be also done to depend only on the Scaling of the Opposite-Spin (SOS) term, thus neglecting same-spin correlation and rerunning the parameterization. Due to the particularities of this electron correlation correction for excited-states, up to four additional parameters need to be determined for these applications: ${ }^{79}$ same-spin indirect term $\left(c_{T}^{S S}\right)$, opposite-spin indirect term $\left(c_{T}^{O S}\right)$, same-spin direct term $\left(c_{U}^{S S}\right)$, and opposite-spin direct term $\left(c_{U}^{O S}\right)$.

\subsection{Justification of the choice of the expressions}

Having explained the different routes followed to develop (most of the existing) double-hybrid density functionals, we will thus employ here the following set of methods to systematically assess the effect of each of the methodological contributions so far presented:

- Configuration Interactions (CI) with Singles (S) and partially introduced Doubles (D) substitutions: CIS, CIS(D), ${ }^{79}$ SCS-CIS(D), ${ }^{80}$ and SOSCIS(D). ${ }^{80}$

- Standard double-hybrid density functionals: B2-PLYP $,{ }^{81} \mathrm{PBE}-\mathrm{QIDH},{ }^{60}$ and PBE0-2. ${ }^{82}$

- Spin-scaled double-hybrid density functionals: SCS/SOS-B2PLYP21, ${ }^{78}$ SCS-PBE-QIDH ${ }^{78}$ and SOS-PBE-QIDH. ${ }^{78}$

- Range-separated double-hybrid density functionals: $\omega \mathrm{B} 2-\mathrm{PLYP}^{64}$ and RSX-QIDH. ${ }^{65}$

- Spin-scaled range-separated double-hybrid density functionals: SCS/SOS$\omega$ B2PLYP, ${ }^{78}$ SCS-RSX-QIDH, ${ }^{78}$ and SOS-RSX-QIDH. ${ }^{78}$ 
Hybrid density functionals will not be explored, since it is well-known from previous studies how these expression in any of their forms are unable to provide negative $\Delta E_{S T}$ values for these compounds. ${ }^{32,33}$ The reason is the lack of explicit double excitations, as it happens for CIS too, which would need to go beyond standard TD-DFT treaments. ${ }^{83-86}$ Note also the existing similarities between CIS(D) and any double-hybrid extension for excited states, with the latter depending critically on the $a_{c}$ weight, once electron correlation effects beyond single-excitations are introduced even approximately. We will also explore the SCS-CIS(D) and SOS-CIS(D) variants, to see if some improvements are also found with respect to CIS(D).

We also provide some additional explanation to better understand this choice of double-hybrid functionals, with specific information about the set of functionals gathered in Table 1. There is not a SCS-B2PLYP or SCS$\omega$ B2-PLYP version since the SCS fit collapsed to the SOS variant, ${ }^{78}$ and thus the original authors consequently dubbed them as SCS/SOS-B2PLYP or SCS/SOS- $\omega$ B2-PLYP. Additionally, the reparameterization of the original B2-PLYP functional for excited states led to a version renamed now as SCS/SOS-B2PLYP21 ${ }^{78}$ to distinguish from a previously developed one. We have not considered other related double-hybrid functionals with $a_{c}$ values close to the ones explored here (e.g. B2GP-PLYP ${ }^{57}$ has a $a_{c}=0.35$ value, close to that of PBE-QIDH) neither other models with low $a_{c}$ values (vide infra) such as e.g. PBE0-DH ${ }^{59}$ for which $a_{c}=1 / 8$.

\subsection{Computational details}

The ground-state $\left(S_{0}\right)$ geometry of all the compounds was optimized with the B97-3c method, ${ }^{87}$ with all-real $3 N-6$ vibrational frequencies obtained in all cases. The vertical excitation energies are thus calculated at those global minimum geometries, and for the lowest excited states of singlet $\left(S_{1}\right)$ and 
triplet $\left(T_{1}\right)$ multiplicity, denoted respectively as $S_{1} \leftarrow S_{0}$ and $T_{1} \leftarrow S_{0}$. Due to the rigidity of these systems, the difference between vertical and adiabatic excitation energies is known to be minimal. ${ }^{24}$ The singlet-triplet energy difference is always calculated as $\Delta E_{S T}=E\left(S_{1} \leftarrow S_{0}\right)-E\left(T_{1} \leftarrow S_{0}\right)$, normally being $\Delta E_{S T}>0$ unless an inversion of $S_{1}$ and $T_{1}$ excited-state energies is found for which $\Delta E_{S T}$ will be found negative. The double-hybrid methods are employed within the standard linear-response full TD-DFT method and under the Tamm-Dancoff approximation ${ }^{88}$ or TDA-DFT. Note that the TDA-DFT was found before to give slightly more accurate $T_{1} \leftarrow S_{0}$ excitation energies for locally-excited excitations, ${ }^{15}$ but its use was restricted to hybrid expressions in the linear-response regime, so that we will also assess if some difference arises here. The def2-TZVP basis set ${ }^{89}$ was used for all the calculations unless otherwise noticed, with the auxiliary def2/JK and def2-TZVP/C basis sets. ${ }^{90}$ Note that for double-hybrid TD(A)-DFT applications, e.g with the B2-PLYP functional, a previous study ${ }^{77}$ found a difference of only $0.01 \mathrm{eV}$ for excitation energies calculated with the def2-TZVP compared with the larger def2-QZVP basis set, which is thus not expected to significantly alter the conclusions to be reached here at least qualitatively. However, we will also complementarily study if some difference significant arises here with smaller (e.g. def2-SVP) and larger (e.g. def2-QZVP) basis sets. All the CIS(D) and double-hybrid density functional calculations are done with the ORCA 5.0 package. ${ }^{91} \mathrm{Fi}$ nally, some SCS-CC2 values, considered here to be a single-reference method to allow a more homogeneous comparison, were also calculated in this work with the TURBOMOLE 7.4 package. ${ }^{92}$ 


\section{Analysis of the results}

\section{$4.12 \mathrm{~T}-\mathrm{N}, 2 \mathrm{~T}-4 \mathrm{~N}$, and $2 \mathrm{~T}-7 \mathrm{~N}$ systems}

\subsubsection{Wavefunction methods}

Table 2 presents first the results for the set of $2 \mathrm{~T}-\mathrm{N}, 2 \mathrm{~T}-4 \mathrm{~N}$, and $2 \mathrm{~T}-$ $7 \mathrm{~N}$ systems, for which a large number of reference values by previously applied wavefunction methods are available. Note that some studies [DLPNOSTEOM-CCSD, NEVPT2, SCS-CC2, and SCS-ADC(2)] used the def2-TZVP basis set, as done here, but others [EOM-CCSD, $\operatorname{ADC}(2)$, and $\mathrm{ADC}(3)] \mathrm{em}-$ ployed the cc-pVDZ basis set instead. However, we have preferred to consider all of these values as a guide, to clearly show that in all cases a $\Delta E_{S T}<0$ value was obtained by any combination of method and basis set. Comparing all of these wavefunction results, we note an overestimation of values (at least for 2T-N and $2 \mathrm{~T}-7 \mathrm{~N}$ ) by the DLPNO-STEOM-CCSD method compared with EOM-CCSD, ADC(3), or NEVPT2. If we thus discard those DLPNOSTEOM-CCSD values, we could obtain the averaged value (simply as a qualitative rule-of-thumb) of all the other methods mentioned. This brackets the $\Delta E_{S T}$ values (in $\mathrm{eV}$ ) as -0.14 (betweem -0.04 and -0.23 ), -0.16 (between -0.08 and -0.18 ), and -0.24 (between -0.11 and -0.38 ) for $2 \mathrm{~T}-\mathrm{N}, 2 \mathrm{~T}-4 \mathrm{~N}$, and $2 \mathrm{~T}-7 \mathrm{~N}$ systems, respectively. Note that, roughly speaking, the $\Delta E_{S T}$ becomes slightly more negative as the number of $\mathrm{N}$ atoms increases.

First of all, looking at the CIS-based methods, the simplest CIS treatment is unable to predict the excited-state energy inversion, as also reported previously, while CIS(D) does it although providing larger $\Delta E_{S T}$ values $(-0.28$, -0.29 , and $-0.52 \mathrm{eV}$ for $2 \mathrm{~T}-\mathrm{N}, 2 \mathrm{~T}-4 \mathrm{~N}$, and $2 \mathrm{~T}-7 \mathrm{~N}$, respectively, see Table 2 ) than most of the other wavefunction methods included in Table 2 and taken as reference values. The SCS-CIS(D) [SOS-CIS(D)] correction still decreases the latter $\Delta E_{S T}$ value by $-0.12,-0.11$, and $-0.17 \mathrm{eV}(-0.34,-0.27$, and $-0.38 \mathrm{eV}$ ) for $2 \mathrm{~T}-\mathrm{N}, 2 \mathrm{~T}-4 \mathrm{~N}$, and $2 \mathrm{~T}-7 \mathrm{~N}$ systems, respectively. This discrep- 
ancy, with respect to the set of values included as references, can be attributed to a too pronounced stabilization of the $S_{1}$ state of these systems: note that the large underestimation of the $S_{1} \leftarrow S_{0}$ excitation energy compared with the $T_{1} \leftarrow S_{0}$ seems to indicate for the systems tackled here displayed an unbalanced description of correlation effects by spin-scaled CIS(D) under their current parameterization.

\subsubsection{Double-hybrid density functionals}

We will first comment on the B2-PLYP and PBE-QIDH results, inspecting again Table 2, for which very similar $\Delta E_{S T}>0\left(\Delta E_{S T}<0\right)$ values are found for $2 \mathrm{~T}-\mathrm{N}$ and $2 \mathrm{~T}-4 \mathrm{~N}(2 \mathrm{~T}-7 \mathrm{~N})$ systems: These two methods are thus not able to give the excited-state energy inversion for $2 \mathrm{~T}-\mathrm{N}$ and $2 \mathrm{~T}-4 \mathrm{~N}$. Note that the corresponding range-separation extension, $\omega \mathrm{B} 2-\mathrm{PLYP}$ and RSX-QIDH, respectively, still keeps the wrong sign of $\Delta E_{S T}$ for $2 \mathrm{~T}-\mathrm{N}$ and $2 \mathrm{~T}-4 \mathrm{~N}$, and reverse it for $2 \mathrm{~T}-7 \mathrm{~N}$. Therefore, range-separation alone does not help here, since long-range exchange effects do not drive this excited-state energy inversion. ${ }^{46}$ On the other hand, the PBE0-2 model is able to predict the correct $\Delta E_{S T}<0$ values for the three molecules selected, thanks to its higher $a_{c}$ value (compared to that of B2-PLYP and PBE-QIDH) weigthing the second-order perturbation term, whose application to excited states is highly benefitial here. If we analyze next the set of SCS- or SOS-based functionals, we can see how $\Delta E_{S T}<0$ values are predicted for 2T-N at the SCS/SOS-B2PLYP21, SCS/SOS- $\omega$ B2PLYP, SCS-PBE-QIDH, SOS-PBE-QIDH, and SOS-RSX-QIDH levels (and thus with all the variants with the exception of SCS-RSX-QIDH). However, for $2 \mathrm{~T}-4 \mathrm{~N}$ only SOS-PBE-QIDH gives a $\Delta E_{S T}<0$ value (apparently too low) with all other methods wrongly providing $\Delta E_{S T}>0$, which clearly shows how challenging is to calculate this excited-state energy inversion typical of this system. We thus recommend to include it on any benchmark study to be further performed. 


\section{$4.2 \quad 2 \mathrm{~T}-3 \mathrm{~N}, 2 \mathrm{~T}-4_{\mathrm{bis}} \mathrm{N}, 2 \mathrm{~T}-5 \mathrm{~N}$, and $2 \mathrm{~T}-5_{\mathrm{bis}} \mathrm{N}$ systems}

\subsubsection{Wavefunction methods}

We will describe next the CIS, CIS(D), SCS-CIS(D), and SOS-CIS(D) results gathered in Table 3 for the new set of in silico discovered but not-yetsynthesized azaphenalene systems. For this set of molecules, only EOM-CCSD results are available from the original study, but consistently showing again $\Delta E_{S T}<0$ values between -0.03 and $-0.08 \mathrm{eV}$, and thus slightly lower than those found for the previous $2 \mathrm{~T}-\mathrm{N}, 2 \mathrm{~T}-4 \mathrm{~N}$, and $2 \mathrm{~T}-7 \mathrm{~N}$ systems at the same level of theory. Correspondingly, CIS(D) overestimates the values by $0.3-0.4$ with respect to EOM-CCSD, leading thus to too negative $\Delta E_{S T}$ energy difference, with a more pronounced overestimation upon application of the SCS or SOS scaling. Once again, we find a large underestimation of the $S_{1} \leftarrow S_{0}$ excitation energies which translates to those too negative $\Delta E_{S T}$ values.

\subsubsection{Double-hybrid density functionals}

The results of all the assessed double-hybrid density functionals are also presented in Table 3. Strikingly, none of BLYP-based functionals (be B2PLYP, $\omega \mathrm{B} 2-\mathrm{PLYP}$ or the spin-scaled SCS/SOS-B2PLYP21 or SCS/SOS- $\omega$ B2PLYP versions) was able to provide now the correct $\Delta E_{S T}<0$ value, although SCS/SOS-B2PLYP21 or SCS/SOS- $\omega$ B2PLYP led to very low $\Delta E_{S T}$ values from quasi-isoenergetic $S_{1}$ and $T_{1}$ excited states. On the other hand, some members of the PBE-based family of functionals (SCS-PBE-QIDH, SOS-PBEQIDH, SOS-RSX-QIDH, and PBE0-2) agree qualitatively and quantitatively with EOM-CCSD results, as it also happened for the previous 2T-N, 2T-4N, and $2 \mathrm{~T}-7 \mathrm{~N}$ systems. 


\subsection{The case of HAP-3MF}

We finally discuss the case of the real-world dye dubbed as HAP-3MF, for which the $\mathrm{ADC}(2)$ and SCS-CC2 calculations predicted a $\Delta E_{S T}<0$ value in agreement with experimental findings. Although results with a large (def2TZVP) and a moderate (def2-SVP) basis set are calculated here, we will analyze first those obtained with the def2-TZVP one accordingly to previous systems. The effect of using another basis set will be later analyzed. As we can easily see from Table 4, all the (D)-corrected methods now led to an excited-state energy inversion, with SCS/SOS- $\omega$ B2PLYP, SCS-PBE-QIDH, SOS-PBE-QIDH, SCS-RSX-QIDH, and PBE0-2 agreeing within $0.1 \mathrm{eV}$ with respect to the SCS-CC2 values. The $\omega$ B2-PLYP and RSX-QIDH methods still predicted a very small but positive value. Note also that the good results obtained by SCS-PBE-QIDH, SOS-PBE-QIDH, and PBE0-2 double-hybrid functionals are not due to some error compensation, since both $S_{1} \leftarrow S_{0}$ and $T_{1} \leftarrow S_{0}$ excitation energies reasonably agree well between them and with respect to SCS-CC2 results although slightly underestimated.

\section{Discussion of the results}

\subsection{Basis set effects}

Before continuing with the detailed analysis of the results, we would also like to analyze if basis set effects could bring any qualitative difference to alter the overall performance of the methods. First, the basis set effect is not very pronounced going from the def2-TZVP to the larger def2-QZVP, which it was assessed with the PBE-QIDH functional as a test case for the molecular set of $2 \mathrm{~T}-\mathrm{N}, 2 \mathrm{~T}-3 \mathrm{~N}, 2 \mathrm{~T}-4 \mathrm{~N}, 2 \mathrm{~T}-4_{\mathrm{bis}} \mathrm{N}, 2 \mathrm{~T}-5 \mathrm{~N}, 2 \mathrm{~T}-5_{\mathrm{bis}} \mathrm{N}$, and $2 \mathrm{~T}-7 \mathrm{~N}$ systems. Interestingly, the $S_{1} \leftarrow S_{0}$ and $T_{1} \leftarrow S_{0}$ excitation energies changed as much as 0.06 and $0.05 \mathrm{eV}$, respectively, with a completely negligible influence on the corresponding $\Delta E_{S T}$ values up to $0.01 \mathrm{eV}$. Therefore, extending the basis set 
is not expected to significantly influence the conclusions reached here about the performance of a double-hybrid density functional.

Additionally, note that the cost-effective def2-SVP basis set was also used for the large HAP-3MF dye. We did that taking into account its possible use in data-driven massive studies of real-world dyes, for which a less costly basis set than def2-TZVP could be needed for methods scaling with system size $(N)$ as $O\left(N^{5}\right)$ (or beyond). We can see in Table 4 how $\left|\Delta E_{S T}\right|$ is modified by up to $0.05 \mathrm{eV}$ but with most of the assessed methods still giving negative values. However, its impact on the SCS-CC2 method is rather significant on $S_{1} \leftarrow S_{0}$ excitation energies. Therefore, for preliminary or screening studies using double-hybrid methods, the use of this basis set should not be discarded since it is not expected to significantly modify the conclusions if a recommended functional (vide infra) is used with it.

\subsection{TDA-DFT vs. TD-DFT results}

Since the Tamm-Dancoff approximation has been extensively used for excited-state studies with great success, ${ }^{93,94}$ and particularly for TADF emitters to also improve (even slightly) the $T_{1} \leftarrow S_{0}$ excitation energy calculated by hybrid density functionals, ${ }^{15}$ we have repeated the whole set of TD-DFT calculations with this approximation (TDA-DFT). The results are presented in Tables 5-7, analogously to Tables 2-4. The use of TDA-DFT increases both the $S_{1} \leftarrow S_{0}$ and $T_{1} \leftarrow S_{0}$ excitation energies, but not in the same amount which translates into some variations of the corresponding $\Delta E_{S T}$ values. Generally speaking, $T_{1} \leftarrow S_{0}$ are slightly more affected than $S_{1} \leftarrow S_{0}$ excitation energies, with spin-scaling functionals more proned to feel that effect. $S_{1} \leftarrow S_{0}$ values can vary up to $0.15 \mathrm{eV}$ (e.g. with the SCS-RSX-QIDH or SOS-RSX-QIDH functionals and for all the set of molecules) while $T_{1} \leftarrow S_{0}$ can differ up to $0.20 \mathrm{eV}$ (again for the same SCS-RSX-QIDH and SOS-RSX- 
QIDH cases taken as example). The variations of $\Delta E_{S T}$ values span a range of up to $0.3 \mathrm{eV}$, meaning that the maximum decrease (increase) was $-0.16(0.12)$ eV, again with SCS-RSX-QIDH and SOS-RSX-QIDH, but with some functionals like B2-PLYP, PBE-QIDH, SCS-PBE-QIDH or SOS-PBE-QIDH less affected, showing variations (in $\mathrm{eV}$ ) comprised between $-0.01 / 0.03,-0.05 / 0.00$, $-0.01 / 0.04$, and $0.00 / 0.05$, respectively.

\subsection{Global overall performances}

Since it is clear that for all the systems selected a $\Delta E_{S T}<0$ value is predicted by wavefunction methods, consistently with other experimental evidences, ${ }^{26,27,34}$ we consider whether a double-hybrid density functional could reproduce that excited-state energy inversion or not, independently of the final numerical values. Figure 4 thus presents the number of double-hybrid expressions able to behave correctly (at least qualitatively) with respect to the total number of molecules, and with the full TD-DFT scheme: PBE0-2, SCS-PBE-QIDH, and SOS-PBE-QIDH are able to do it for all the systems, closely followed by the SOS-RSX-QIDH method. On the other hand, Figure 4 also displays the number of double-hybrid expressions able to predict the excite-state energy inversion under the Tamm-Dancoff approximation, to graphically appreciate better the differences with respect to the full TD-DFT treatment: SOS-PBE-QIDH and SOS-RSX-QIDH are now the best performers, closely followed by PBE0-2 and SCS-PBE-QIDH.

Overall, spin-scaled PBE-QIDH-based methods seem to behave more accurately than B2-PLYP-based ones, independently if we invoke the TDA-DFT or the full TD-DFT treatment. A notable exception is PBE0-2 for which no scaling is needed in any case. We relate (vide infra) this behaviour with the large $a_{c}$ weight of its perturbative term, compared with B2-PLYP or PBE-QIDH expressions, which in the case of PBE-QIDH is compensated by the spin-scaling 
done. Additionally, these methods are more accurate than spin-scaled CIS(D), which led to larger deviations not only for $\Delta E_{S T}$ but also for individual excitation energies. On the other hand, range-separation is therefore not needed for this kind of molecules, contrarily to other truly charge-transfer systems. ${ }^{95,96}$

\subsection{Individual overall performances}

We analyze next, after establishing a rule-of-thumb for the selection of a double-hybrid density functional, the accuracy of each method for the $S_{1} \leftarrow$ $S_{0}, T_{1} \leftarrow S_{0}$, and $\Delta E_{S T}$ energies. The 'Mean-Signed Deviation' (MSD), the 'Mean Absolute Deviation' (MAD), and the 'Root Mean-Squared Deviation' (RMSD) will obtained for all the methods assessed as: MSD = $\frac{1}{N} \sum_{i}^{N} x_{i}, \mathrm{MAD}=\frac{1}{N} \sum_{i}^{N}\left|x_{i}\right|$, and RMSD $=\sqrt{\frac{1}{N} \sum_{i}^{N} x_{i}^{2}}$, with $N=8$, $x_{i}=\Omega_{i}^{\text {calculated }}-\Omega_{i}^{\mathrm{SCS}-\mathrm{CC} 2}$, all values obtained with the def2-TZVP basis set considered as accurate enough for all the systems. Table 8 presents these values at the full TD-DFT level. For the $S_{1} \leftarrow S_{0}$ excitation energies, the best performer is SCS/SOS- $\omega$ B2PLYP with a RMSD of only $0.06 \mathrm{eV}$, closely followed by PBE-QIDH and PBE0-2 with a value of $0.09 \mathrm{eV}$. However, the accuracy of SCS/SOS- $\omega$ B2PLYP and PBE-QIDH methods for the $T_{1} \leftarrow S_{0}$ excitation energies is not so good, with RMSD values of 0.26 and $0.22 \mathrm{eV}$, respectively, thus explaining their ranking in the global assessment done in the previous section. PBE0-2 does it well again, with a RMSD of $0.13 \mathrm{eV}$ and thus comparable to that achieved for $S_{1} \leftarrow S_{0}$ excitation energies. The best performer here is the SCS/SOS-B2PLYP21 method with a value as small as 0.05 $\mathrm{eV}$, smaller than that obtain at this level for the $S_{1} \leftarrow S_{0}$ excitation energies $(0.25 \mathrm{eV})$ which is somehow atypical and should be further investigated. Finally, when looking at the $\Delta E_{S T}$ energy differences, a RMSD around $0.1 \mathrm{eV}$ is obtained by SCS-PBE-QIDH, SOS-PBE-QIDH, SOS-RSX-QIDH, and PBE02 methods, those behaving globally more accurately in the sense of providing a $\Delta E_{S T}<0$ value for all the systems. In this regard, it is therefore highly 
advised to select a functional able to perform equally well on both $S_{1} \leftarrow S_{0}$ and $T_{1} \leftarrow S_{0}$ excitation energies, which would translate into accurate $\Delta E_{S T}$ predictions. This statement can be observed e.g. looking at the SOS-PBEQIDH values: a RMSD of $0.09 \mathrm{eV}$ for $\Delta E_{S T}$ (and actually the lowest found among all the methods) arises from reasonably low RMSD values for both $S_{1} \leftarrow S_{0}(0.18 \mathrm{eV})$ and $T_{1} \leftarrow S_{0}(0.22 \mathrm{eV})$ excitation energies, thus showing a robust performance.

\section{On the decisive role of $a_{c}$}

We remind that the $a_{c} \Delta(\mathrm{D})$ term is in fact crucial to correct the excitation energies $(\Omega)$ obtained by any hybrid density functional in the form $\Omega^{\prime}=\Omega+a_{c} \Delta(\mathrm{D})$, which are known to not always lead to $\Delta E_{S T}>0$ (wrong) values. We will denote in the following as B2-PLYP(SCF), PBE-QIDH(SCF), and PBE0-2(SCF) the underlying hybrid functionals giving the $\Omega$ excitation energies, to distinguish from the full B2-PLYP, PBE-QIDH, and PBE0-2 double-hybrid expressions. Actually, given the accuracy of the PBE0-2 model, we exemplify next the $\Delta E_{S T}$ values obtained from the $\Omega$-only $S_{1} \leftarrow S_{0}$ and $T_{1} \leftarrow S_{0}$ excitation energies by PBE0-2(SCF): 0.48, 1.06, 0.46, 0.62, 0.83, 0.72, 0.74 , and $0.31 \mathrm{ev}$, for the $2 \mathrm{~T}-\mathrm{N}, 2 \mathrm{~T}-4 \mathrm{~N}, 2 \mathrm{~T}-7 \mathrm{~N}, 2 \mathrm{~T}-3 \mathrm{~N}, 2 \mathrm{~T}-4_{\mathrm{bis}} \mathrm{N}, 2 \mathrm{~T}-5 \mathrm{~N}$, and $2 \mathrm{~T}-5_{\text {bis }} \mathrm{N}$ systems, respectively. One can easily see that all of them are positive and largely overestimated. This is not exclusive of PBE0-2, since for PBEQIDH $(\mathrm{SCF})$ one would now find: $0.42,0.86,0.41,0.54,0.68,0.62,0.64$, and $0.28 \mathrm{eV}$, respectively, and thus slightly lower values but still all being positive. Finally, for the B2-PLYP(SCF) functional, these values are: 0.32, 0.61, 0.33, $0.42,0.50,0.47,0.49$, and $0.24 \mathrm{eV}$. Note that this pitfall is known to affect any hybrid functionals of those existing, independently if they are range-separated or not, their composition in terms of exchange and correlation functionals, etc. due to the lack of (D)-like excitations as it has been extensively demon- 
strated before. ${ }^{32,33}$ Figure S1 shows the quantity $\Delta(\mathrm{D})=\frac{\Delta E_{S T}\left(\Omega^{\prime}\right)-\Delta E_{S T}(\Omega)}{a_{c}}$ which spans between 1-2 eV among the compounds, although not significantly differing between the three models (B2-PLYP, PBE-QIDH, and PBE0-2) selected for this analysis.

Indeed, that leads the responsability of final values to the marked influence of the $a_{c} \Delta(\mathrm{D})$ contribution, or more particularly speaking the value of $a_{c}$, which makes the difference from (wrong) $\Delta E_{S T}>0$ to (right) $\Delta E_{S T}<0$ values in these systems. That contribution is known to be an individual quantity for each functional, molecule, and excited state, which in fact determines the final quality of an excitation energy calculated by a double-hybrid density functional. ${ }^{97-99}$ Figure 5 presents the magnitude of that term for the standard B2-PLYP, PBE-QIDH, and PBE0-2 expressions, which differ in their $a_{c}$ weight ( $a_{c}=0.27, a_{c}=1 / 3$, and $a_{c}=1 / 2$, respectively) and for both excitation energies $\left(S_{1} \leftarrow S_{0}\right.$ and $\left.T_{1} \leftarrow S_{0}\right)$ for the whole set of systems. The first observation is how the magnitude of the correction for $S_{1} \leftarrow S_{0}$ excitation energies increase with $a_{c}$, as expected, ranging from -0.24 to $-0.71 \mathrm{eV}$ for B2-PLYP, from -0.26 to $-0.76 \mathrm{eV}$ for PBE-QIDH, and from -0.38 to $-1.09 \mathrm{eV}$ for PBE0-2. Note that: (i) $a_{c}$ values for B2-PLYP and PBE-QIDH are close and smaller than that of PBE0-2, thus explaining the similar range of values found, and why is larger for PBE0-2; and (ii) double excitations contributions are always negative for the $S_{1} \leftarrow S_{0}$ excitation energies, significantly contributing thus to the stabilization of the $S_{1}$ state due to correlation effects. ${ }^{100}$ Turning now to $T_{1} \leftarrow S_{0}$ excitation energies, the variation ranges from 0.06 to $-0.40 \mathrm{eV}$ for B2-PLYP, from 0.22 to $-0.41 \mathrm{eV}$ for PBE-QIDH, and from 0.48 to $-0.57 \mathrm{eV}$ for PBE0-2. These findings are also preserved for each molecule and not only globally.

Let us take the $2 \mathrm{~T}-\mathrm{N}$ case as example to further clarify the impact on this 
correction on the final $\Delta E_{S T}$ values. This was calculated from the $\Omega$ excitation energies by $\mathrm{PBE}-\mathrm{QIDH}(\mathrm{SCF})$ to be $0.415 \mathrm{eV}$, that is without the $a_{c} \Delta(\mathrm{D})$ correction. Since that correction decreases the $S_{1} \leftarrow S_{0}$ excitation energy by $-0.263 \mathrm{eV}$ while the $T_{1} \leftarrow S_{0}$ excitation energy increased by $0.114 \mathrm{eV}$, the new (D)-corrected $\Delta E_{S T}$ value changes to $0.038 \mathrm{eV}$, which is the value entering into Table 2. If we repeat the same calculation for PBE0-2, for which the pristine $\Delta E_{S T}$ value obtained from the $\Omega$ excitation energies was $0.478 \mathrm{eV}$ by PBE0-2(SCF), the (D)-like correction decreases the $S_{1} \leftarrow S_{0}$ excitation energy by $-0.383 \mathrm{eV}$ while the $T_{1} \leftarrow S_{0}$ excitation energy increased by $0.197 \mathrm{eV}$, leading now to a (D)-corrected $\Delta E_{S T}$ value of $-0.102 \mathrm{eV}$. Finally, taken the B2-PLYP(SCF) expression, the $\Omega$ excitation energies led to a $\Delta E_{S T}$ value of $0.318 \mathrm{eV}$, which is changed with a decrease of the $S_{1} \leftarrow S_{0}$ excitation energy by $-0.239 \mathrm{eV}$ and an increase of the $T_{1} \leftarrow S_{0}$ excitation energy by $0.063 \mathrm{eV}$, leading to the final value of $0.02 \mathrm{eV}$ gathered in Table 2. Table 9 shows the magnitude of the $a_{c} \Delta(\mathrm{D})$ correction for all the molecules, and for both excitation energies, for which it can be corroborated its key importance for this challenging situation. That strong dependence on the $a_{c} \Delta(\mathrm{D})$ correction also explains why (exchange) range-separation leads to similar results to standard double-hybrid models.

Table 10 finally gathers the magnitude of the $a_{c} \Delta(\mathrm{D})$ correction for all the molecules, and again for both excitation energies, but now considering the spin-scaled SCS-PBE-QIDH and SOS-PBE-QIDH functionals. If we define the ratio between the magnitude of that correction for spin-scaled and spinunscaled functionals as:

$$
\text { ratio }=\frac{a_{c} \Delta(\mathrm{D})[\mathrm{SCS}-\mathrm{PBE}-\mathrm{QIDH}]}{a_{c} \Delta(\mathrm{D})[\mathrm{PBE}-\mathrm{QIDH}]},
$$

we find a value between 1.3-1.6 for the $S_{1} \leftarrow S_{0}$ excitation energy in the case of SCS-PBE-QIDH. In the case of SOS-PBE-QIDH, that ratio slightly increases to $1.4-1.7$, thus showing in both cases a significant impact on the stabilization 
of the $S_{1}$ state since that contribution is always negative for the $S_{1} \leftarrow S_{0}$ excitation energy. Calculating the same ratio now for the $T_{1} \leftarrow S_{0}$ excitation energy, this becomes 0.8-1.2 (0.9-1.3) for SCS-PBE-QIDH (SOS-PBE-QIDH), being thus not pronounced as for the $S_{1} \leftarrow S_{0}$ excitation energy. The interplay between this asymmetry, in the right direction of stabilizing the $S_{1}$ state by correlation effects, causes the excited-state energy inversion of all the systems in agreement with wavefunction and experimental reference results.

\section{Conclusions}

We have elucidated the reasons for the performance of different doublehybrid exchange-correlation functionals to predict the energy inversion of the lowest singlet $\left(S_{1}\right)$ and triplet $\left(T_{1}\right)$ excited states of organic molecules with envisioned optoelectronic or photocatalytic applications. That violation of Hund's rule manifests in a negative singlet-triplet energy difference, $\Delta E_{S T}$, contrarily to most conjugated systems, which could foster a mechanism to recover the triplet excitons after their formation by spin statistics. That recovery could lead to the design of most efficient electroluminiscent organic devices, after a molecular-driven design with predictive nature. However, the lack of explicit double excitations into standard TD-DFT calculations precluded up to now the systematic use of this methodology for that purpose, and thus double-hybrid exchange-correlation functionals incorporating (partially) those double excitations could become a solid alternative to wavefunction methods for this purpose.

To investigate this performance in more detail, we have systematically applied a set of methods to disentangle if a standard double-hybrid expression (i.e., B2-PLYP, PBE-QIDH, PBE0-2) could lead to negative $\Delta E_{S T}$ values, as well as if exchange range-separation (i.e., $\omega \mathrm{B} 2-\mathrm{PLYP}, \mathrm{RSX}-\mathrm{QIDH}$ ) and/or 
spin-scaling for standard (SCS/SOS-B2PLYP21, SCS-PBE-QIDH, SOS-PBEQIDH) or range-separated (SCS/SOS- $\omega$ B2PLYP, SCS-RSX-QIDH, and SOSRSX-QIDH) expressions could help. Not surprisingly, the key aspect of those exchange-correlation methods assessed was the weight given to the double excitations into the calculations, which is known to be mediated by a coefficient $a_{c}$. The minimum weight needed to obtain negative $\Delta E_{S T}$ values is believed to be at least $a_{c} \geq 0.5$. Complementarily, since correlation and not exchange effects are the reason for that excited-state energy inversion, exchange rangeseparation did not bring any advantage with respect to standard double-hybrid expressions. Finally, spin-scaling was able to recover the negative $\Delta E_{S T}$ values, even for some of those functionals unable initially to do it, after scaling the correlation contribution by a factor sufficiently high (e.g. $C_{T}^{O S}$ and $C_{U}^{O S} \geq 0.5$ ).

As a computational recipe to choose one or another double-hybrid functional among all the existing expressions, we recommend to use PBE0-2, SOSPBE-QIDH, SCS-PBE-QIDH, and SOS-RSX-QIDH (or some other comparable) methods, which fit with the recommendations about the $a_{c}$ and $C_{T}^{O S}$ and $C_{U}^{O S}$ values needed. If at least two of these expressions are able to predict a negative $\Delta E_{S T}$ value, it could be robustly inferred that the molecule thus exhibits the excited-state energy inversion. The use of a cost-effective basis set (def2-SVP) with respect to a nearly-converged one (def2-TZVP) did not alter the conclusion reached about which method was able to give that negative $\Delta E_{S T}$ values. Additionally, the use or not of the Tamm-Dancoff approximation does not change significantly the list of methods with an acceptable performance. Overall, we hope this study will contribute to accurately tackle the data-driven design of this challenging systems. 


\section{Acknowledgements}

The work in Alicante is supported by the "Ministerio de Ciencia e Innovación" of Spain (Grant No. PID2019-106114GB-I00). Y.O. acknowledges funding by the "Fonds de la Recherche Scientifique-FNRS" under Grant n. F.4534.21 (MIS-IMAGINE). E.B. thanks ANR (Agence Nationale de la Recherche) and CGI (Commissariat à l'Investissement d'Avenir) for their financial support to this work through Labex SEAM (Science and Engineering for Advanced Materials and devices), Grant Nos. ANR-10-LABX-096 and ANR-18-IDEX-0001. G.R. acknowledges a grant from the "Fonds pour la formation a la Recherche dans l'Industrie et dans l'Agriculture" (F.R.I.A.) of the F.R.S.-F.N.R.S. We acknowledge useful discussions with E. Zysman-Colman (U. St.-Andrews, U.K.), S. Ghosh (Max Planck Institute for Coal Research, Germany), and R. Pollice and A. Aspuru-Guzik (U. Toronto, Canada)

\section{Supplementary Material}

In the Supplementary Material we include: (i) Cartesian (XYZ) atomic coordinates (in $\AA$ ) of all the systems considered; (ii) Representation of the quantity $\Delta(\mathrm{D})=\frac{\Delta E_{S T}\left(\Omega^{\prime}\right)-\Delta E_{S T}(\Omega)}{a_{c}}$

\section{Data Availability}

The data that supports the findings of this study are available within the article [and its supplementary material] or are available from the corresponding authors upon reasonable request.

\section{References}

[1] Köhler, A.; Beljonne, D. The singlet-triplet exchange energy in conjugated polymers. Advanced Functional Materials 2004, 14, 11-18. 
[2] Gierschner, J.; Cornil, J.; Egelhaaf, H.-J. Optical bandgaps of $\pi$ conjugated organic materials at the polymer limit: experiment and theory. Advanced Materials 2007, 19, 173-191.

[3] Köhler, A.; Bässler, H. Triplet states in organic semiconductors. Materials Science and Engineering: R: Reports 2009, 66, 71-109.

[4] Liu, S.; Langenaeker, W. Hund's multiplicity rule: a unified interpretation. Theoretical Chemistry Accounts 2003, 110, 338-344.

[5] Becke, A. D. Singlet-triplet splittings from the virial theorem and singleparticle excitation energies. The Journal of Chemical Physics 2018, 148, 044112.

[6] Becke, A. D. Communication: Optical gap in polyacetylene from a simple quantum chemistry exciton model. The Journal of Chemical Physics 2018, 149, 081102.

[7] Köhler, A.; Wilson, J. S.; Friend, R. H. Fluorescence and phosphorescence in organic materials. Advanced Materials 2002, 14, 701-707.

[8] Endo, A.; Sato, K.; Yoshimura, K.; Kai, T.; Kawada, A.; Miyazaki, H.; Adachi, C. Efficient up-conversion of triplet excitons into a singlet state and its application for organic light emitting diodes. Applied Physics Letters 2011, 98, 42.

[9] Uoyama, H.; Goushi, K.; Shizu, K.; Nomura, H.; Adachi, C. Highly efficient organic light-emitting diodes from delayed fluorescence. Nature 2012, 492, 234-238.

[10] Yang, X.; Xu, X.; Zhou, G. Recent advances of the emitters for high performance deep-blue organic light-emitting diodes. Journal of Materials Chemistry C 2015, 3, 913-944. 
[11] Wong, M. Y.; Zysman-Colman, E. Purely organic thermally activated delayed fluorescence materials for organic light-emitting diodes. Advanced Materials 2017, 29, 1605444.

[12] Yang, Z.; Mao, Z.; Xie, Z.; Zhang, Y.; Liu, S.; Zhao, J.; Xu, J.; Chi, Z.; Aldred, M. P. Recent advances in organic thermally activated delayed fluorescence materials. Chemical Society Reviews 2017, 46, 915-1016.

[13] Wong, M. Y. et al. Deep-blue oxadiazole-containing thermally activated delayed fluorescence emitters for organic light-emitting diodes. ACS Applied Materials \& Interfaces 2018, 10, 33360-33372.

[14] Liu, Y.; Li, C.; Ren, Z.; Yan, S.; Bryce, M. R. All-organic thermally activated delayed fluorescence materials for organic light-emitting diodes. Nature Reviews Materials 2018, 3, 18020.

[15] Moral, M.; Muccioli, L.; Son, W.-J.; Olivier, Y.; Sancho-García, J.-C. Theoretical rationalization of the singlet-triplet gap in OLEDs materials: impact of charge-transfer character. Journal of Chemical Theory and Computation 2015, 11, 168-177.

[16] Etherington, M. K.; Gibson, J.; Higginbotham, H. F.; Penfold, T. J.; Monkman, A. P. Revealing the spin-vibronic coupling mechanism of thermally activated delayed fluorescence. Nature Communications 2016 , $7,1-7$.

[17] Gibson, J.; Monkman, A. P.; Penfold, T. J. The importance of vibronic coupling for efficient reverse intersystem crossing in thermally activated delayed fluorescence molecules. ChemPhysChem 2016, 17, 2956-2961.

[18] Olivier, Y.; Moral, M.; Muccioli, L.; Sancho-García, J.-C. Dynamic nature of excited states of donor-acceptor TADF materials for OLEDs: how theory can reveal structure-property relationships. Journal of Materials Chemistry C 2017, 5, 5718-5729. 
[19] Olivier, Y.; Yurash, B.; Muccioli, L.; D'Avino, G.; Mikhnenko, O.; Sancho-García, J.-C.; Adachi, C.; Nguyen, T.-Q.; Beljonne, D. Nature of the singlet and triplet excitations mediating thermally activated delayed fluorescence. Physical Review Materials 2017, 1, 075602.

[20] Milián-Medina, B.; Gierschner, J. Computational design of low singlettriplet gap all-organic molecules for OLED application. Organic Electronics 2012, 13, 985-991.

[21] Hatakeyama, T.; Shiren, K.; Nakajima, K.; Nomura, S.; Nakatsuka, S.; Kinoshita, K.; Ni, J.; Ono, Y.; Ikuta, T. Ultrapure blue thermally activated delayed fluorescence molecules: efficient HOMO-LUMO separation by the multiple resonance effect. Advanced Materials 2016, 28, $2777-2781$.

[22] Nakatsuka, S.; Gotoh, H.; Kinoshita, K.; Yasuda, N.; Hatakeyama, T. Divergent Synthesis of Heteroatom-Centered 4, 8, 12-Triazatriangulenes. Angewandte Chemie International Edition 2017, 56, 5087-5090.

[23] Matsui, K.; Oda, S.; Yoshiura, K.; Nakajima, K.; Yasuda, N.; Hatakeyama, T. One-shot multiple borylation toward BN-doped nanographenes. Journal of the American Chemical Society 2018, 140, $1195-1198$.

[24] Pershin, A.; Hall, D.; Lemaur, V.; Sancho-García, J.-C.; Muccioli, L.; Zysman-Colman, E.; Beljonne, D.; Olivier, Y. Highly emissive excitons with reduced exchange energy in thermally activated delayed fluorescent molecules. Nature Communications 2019, 10, 1-5.

[25] Sanz-Rodrigo, J.; Olivier, Y.; Sancho-García, J.-C. Computational studies of molecular materials for unconventional energy conversion: The challenge of light emission by thermally activated delayed fluorescence. Molecules 2020, 25, 1006. 
[26] Leupin, W.; Wirz, J. Low-lying electronically excited states of

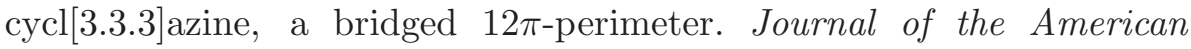
Chemical Society 1980, 102, 6068-6075.

[27] Leupin, W.; Magde, D.; Persy, G.; Wirz, J. 1,4,7-Triazacycl[3.3.3]azine: basicity, photoelectron spectrum, photophysical properties. Journal of the American Chemical Society 1986, 108, 17-22.

[28] Audebert, P.; Kroke, E.; Posern, C.; Lee, S.-H. State of the Art in the Preparation and Properties of Molecular Monomeric s-Heptazines: Syntheses, Characteristics, and Functional Applications. Chemical Reviews 2021, 121, 2515-2544.

[29] Li, J.; Tao, L.; Wang, Y.; Yao, Y.; Guo, Q. Heptazine-Based $\pi$ Conjugated Materials for Light-Emitting. Frontiers in Chemistry 2021, 9,482 .

[30] Kimber, P.; Plasser, F. Toward an understanding of electronic excitation energies beyond the molecular orbital picture. Physical Chemistry Chemical Physics 2020, 22, 6058-6080.

[31] Eng, J.; Penfold, T. J. Open questions on the photophysics of thermally activated delayed fluorescence. Communications Chemistry 2021, 4, 14.

[32] Ricci, G.; San-Fabián, E.; Olivier, Y.; Sancho-García, J.-C. Singlettriplet excited-state inversion in heptazine and related molecules: assessment of TD-DFT and ab initio methods. ChemPhysChem 2021, 22, $553-560$.

[33] de Silva, P. Inverted singlet-triplet gaps and their relevance to thermally activated delayed fluorescence. The Journal of Jhysical Chemistry Letters 2019, 10, 5674-5679. 
[34] Ehrmaier, J.; Rabe, E. J.; Pristash, S. R.; Corp, K. L.; Schlenker, C. W.; Sobolewski, A. L.; Domcke, W. Singlet-triplet inversion in heptazine and in polymeric carbon nitrides. The Journal of Physical Chemistry A 2019, 123, 8099-8108.

[35] Sandoval-Salinas, M. E.; Carreras, A.; Casanova, D. Triangular graphene nanofragments: open-shell character and doping. Physical Chemistry Chemical Physics 2019, 21, 9069-9076.

[36] Schwabe, T.; Grimme, S. Theoretical thermodynamics for large molecules: walking the thin line between accuracy and computational cost. Accounts of Chemical Research 2008, 41, 569-579.

[37] Zhang, Y.; Xu, X.; Goddard, W. A. Doubly hybrid density functional for accurate descriptions of nonbond interactions, thermochemistry, and thermochemical kinetics. Proceedings of the National Academy of Sciences 2009, 106, 4963-4968.

[38] Grimme, S. Density functional theory with London dispersion corrections. Wiley Interdisciplinary Reviews: Computational Molecular Science 2011, 1, 211-228.

[39] Zhang, I. Y.; Su, N. Q.; Brémond, E. A.; Adamo, C.; Xu, X. Doubly hybrid density functional xDH-PBE0 from a parameter-free global hybrid model PBE0. The Journal of Chemical Physics 2012, 136, 174103.

[40] Sancho-García, J.-C.; Adamo, C. Double-hybrid density functionals: merging wavefunction and density approaches to get the best of both worlds. Physical Chemistry Chemical Physics 2013, 15, 14581-14594.

[41] Goerigk, L.; Grimme, S. Double-hybrid density functionals. Wiley Interdisciplinary Reviews: Computational Molecular Science 2014, 4, 576600 . 
[42] Brémond, E.; Ciofini, I.; Sancho-García, J. C.; Adamo, C. Nonempirical double-hybrid functionals: An effective tool for chemists. Accounts of chemical research 2016, 49, 1503-1513.

[43] Bremond, E.; Savarese, M.; Pérez-Jiménez, Á. J.; Sancho-García, J. C.; Adamo, C. Systematic improvement of density functionals through parameter-free hybridization schemes. The Journal of Physical Chemistry Letters 2015, 6, 3540-3545.

[44] Pios, S.; Huang, X.; Sobolewski, A. L.; Domcke, W. Triangular boron carbon nitrides: An unexplored family of chromophores with unique properties for photocatalysis and optoelectronics. Physical Chemistry Chemical Physics 2021, 23, 12698-12675.

[45] Bhattacharyya, K. Can TDDFT render the electronic excited states ordering of Azine derivative? A closer investigation with DLPNOSTEOM-CCSD. Chemical Physics Letters 2021, r79, 138827.

[46] Sanz-Rodrigo, J.; Ricci, G.; Olivier, Y.; Sancho-Garcia, J.-C. Negative Singlet-Triplet Excitation Energy Gap in Triangle-Shaped Molecular Emitters for Efficient Triplet Harvesting. The Journal of Physical Chemistry A 2021, 125, 513-522.

[47] Pollice, R.; dos Passos Gomes, G.; Aldeghi, M.; Hickman, R. J.; Krenn, M.; Lavigne, C.; Lindner-D’Addario, M.; Nigam, A.; Ser, C. T.; Yao, Z.; Aspuru-Guzik, A. Data-driven strategies for accelerated materials design. Accounts of Chemical Research 2021, 54, 849-860.

[48] Pollice, R.; Friederich, P.; Lavigne, C.; dos Passos Gomes, G.; AspuruGuzik, A. Organic molecules with inverted gaps between first excited singlet and triplet states and appreciable fluorescence rates. Matter 2021, 4, 1654-1682.

[49] Li, J.; Nakagawa, T.; MacDonald, J.; Zhang, Q.; Nomura, H.; 
Miyazaki, H.; Adachi, C. Highly efficient organic light-emitting diode based on a hidden thermally activated delayed fluorescence channel in a heptazine derivative. Advanced Materials 2013, 25, 3319-3323.

[50] Li, J.; Zhang, Q.; Nomura, H.; Miyazaki, H.; Adachi, C. Thermally activated delayed fluorescence from ${ }^{3} \mathrm{n} \pi^{*}$ to ${ }^{1} \mathrm{n} \pi^{*}$ up-conversion and its application to organic light-emitting diodes. Applied Physics Letters 2014, 105, 98_1.

[51] Li, J.; Nomura, H.; Miyazaki, H.; Adachi, C. Highly efficient exciplex organic light-emitting diodes incorporating a heptazine derivative as an electron acceptor. Chemical Communications 2014, 50, 6174-6176.

[52] Sobolewski, A. L.; Domcke, W. Are Heptazine-Based Organic LightEmitting Diode Chromophores Thermally Activated Delayed Fluorescence or Inverted Singlet-Triplet Systems? The Journal of Physical Chemistry Letters 2021, 12, 6852-6860.

[53] Head-Gordon, M.; Rico, R. J.; Oumi, M.; Lee, T. J. A doubles correction to electronic excited states from configuration interaction in the space of single substitutions. Chemical Physics Letters 1994, 219, 21-29.

[54] Grimme, S.; Neese, F. Double-hybrid density functional theory for excited electronic states of molecules. The Journal of Chemical Physics 2007, 127, 154116.

[55] Ottochian, A.; Morgillo, C.; Ciofini, I.; Frisch, M. J.; Scalmani, G.; Adamo, C. Double hybrids and time-dependent density functional theory: An implementation and benchmark on charge transfer excited states. Journal of Computational Chemistry 2020, 41, 1242-1251.

[56] Schwabe, T.; Grimme, S. Towards chemical accuracy for the thermodynamics of large molecules: new hybrid density functionals including 
non-local correlation effects. Physical Chemistry Chemical Physics 2006, 8, 4398-4401.

[57] Karton, A.; Tarnopolsky, A.; Lamére, J.-F.; Schatz, G. C.; Martin, J. M. Highly accurate first-principles benchmark data sets for the parametrization and validation of density functional and other approximate methods. Derivation of a robust, generally applicable, double-hybrid functional for thermochemistry and thermochemical kinetics. The Journal of Physical Chemistry A 2008, 112, 12868-12886.

[58] Toulouse, J.; Sharkas, K.; Brémond, E.; Adamo, C. Communication: Rationale for a new class of double-hybrid approximations in densityfunctional theory. The Journal of Chemical Physics 2011, 135, 101102.

[59] Brémond, E.; Adamo, C. Seeking for parameter-free double-hybrid functionals: the PBE0-DH model. The Journal of Chemical Physics 2011, 135, 024106.

[60] Brémond, É.; Sancho-García, J.; Pérez-Jiménez, Á.; Adamo, C. Communication: double-hybrid functionals from adiabatic-connection: the QIDH model. The Journal of Chemical Physics 2014, 141, 031101.

[61] Tozer, D.; Handy, N. On the determination of excitation energies using density functional theory. Physical Chemistry Chemical Physics 2000, 2, 2117-2121.

[62] Tozer, D. Relationship between long-range charge-transfer excitation energy error and integer discontinuity in Kohn-Sham theory. The Journal of Chemical Physics 2003, 119, 12697-12699.

[63] Peach, M. J.; Benfield, P.; Helgaker, T.; Tozer, D. J. Excitation energies in density functional theory: An evaluation and a diagnostic test. The Journal of Chemical Physics 2008, 128, 044118. 
[64] Casanova-Páez, M.; Dardis, M. B.; Goerigk, L. $\omega B 2 P L Y P$ and $\omega$ B2GPPLYP: the first two double-hybrid density functionals with longrange correction optimized for excitation energies. Journal of Chemical Theory and Computation 2019, 15, 4735-4744.

[65] Brémond, E.; Savarese, M.; Pérez-Jiménez, Á. J.; Sancho-García, J. C.; Adamo, C. Range-Separated Double-Hybrid Functional from Nonempirical Constraints. Journal of Chemical Theory and Computation 2018, 14, 4052-4062.

[66] Brémond, É.; Pérez-Jiménez, Á. J.; Sancho-García, J. C.; Adamo, C. Range-separated hybrid density functionals made simple. The Journal of Chemical Physics 2019, 150, 201102.

[67] Brémond, É.; Pérez-Jiménez, Á. J.; Sancho-García, J. C.; Adamo, C. Range-separated hybrid and double-hybrid density functionals: A quest for the determination of the range-separation parameter. The Journal of Chemical Physics 2020, 152, 244124.

[68] Mester, D.; Kállay, M. A simple range-separated double-hybrid density functional theory for excited states. Journal of Chemical Theory and Computation 2021, 17, 927-942.

[69] Zhang, I. Y.; Xu, X. Reaching a uniform accuracy for complex molecular systems: long-range-corrected XYG3 doubly hybrid density functional. The Journal of Physical Chemistry Letters 2013, 4, 1669-1675.

[70] Chan, B.; Goerigk, L.; Radom, L. On the inclusion of post-MP2 contributions to double-Hybrid density functionals. Journal of Computational Chemistry 2016, 37, 183-193.

[71] Grimme, S.; Goerigk, L.; Fink, R. F. Spin-component-scaled electron correlation methods. Wiley Interdisciplinary Reviews: Computational Molecular Science 2012, 2, 886-906. 
[72] Brémond, É.; Savarese, M.; Sancho-García, J. C.; Pérez-Jiménez, Á. J.; Adamo, C. Quadratic integrand double-hybrid made spin-componentscaled. The Journal of Chemical Physics 2016, 144, 124104.

[73] Kozuch, S.; Gruzman, D.; Martin, J. M. DSD-BLYP: A general purpose double hybrid density functional including spin component scaling and dispersion correction. The Journal of Physical Chemistry C 2010, 114, 20801-20808.

[74] Kozuch, S.; Martin, J. M. DSD-PBEP86: in search of the best doublehybrid DFT with spin-component scaled MP2 and dispersion corrections. Physical Chemistry Chemical Physics 2011, 13, 20104-20107.

[75] Kozuch, S.; Martin, J. M. Spin-component-scaled double hybrids: an extensive search for the best fifth-rung functionals blending DFT and perturbation theory. Journal of Computational Chemistry 2013, 34, $2327-2344$.

[76] Santra, G.; Sylvetsky, N.; Martin, J. M. Minimally empirical doublehybrid functionals trained against the GMTKN55 database: revDSDPBEP86-D4, revDOD-PBE-D4, and DOD-SCAN-D4. The Journal of Physical Chemistry A 2019, 123, 5129-5143.

[77] Schwabe, T.; Goerigk, L. Time-dependent double-hybrid density functionals with spin-component and spin-opposite scaling. Journal of Chemical Theory and Computation 2017, 13, 4307-4323.

[78] Casanova-Páez, M.; Goerigk, L. Time-Dependent Long-RangeCorrected Double-Hybrid Density Functionals with Spin-Component and Spin-Opposite Scaling: A Comprehensive Analysis of SingletSinglet and Singlet-Triplet Excitation Energies. Journal of Chemical Theory and Computation 2021, 17, 5165-5186. 
[79] Rhee, Y. M.; Head-Gordon, M. Scaled second-order perturbation corrections to configuration interaction singles: Efficient and reliable excitation energy methods. The Journal of Physical Chemistry A 2007, 111, $5314-5326$.

[80] Goerigk, L.; Grimme, S. Assessment of TD-DFT methods and of various spin scaled CIS (D) and CC2 versions for the treatment of low-lying valence excitations of large organic dyes. The Journal of Chemical Physics 2010, 132, 184103.

[81] Grimme, S. Semiempirical hybrid density functional with perturbative second-order correlation. The Journal of Chemical Physics 2006, 124, 034108.

[82] Chai, J.-D.; Mao, S.-P. Seeking for reliable double-hybrid density functionals without fitting parameters: the PBE0-2 functional. Chemical Physics Letters 2012, 538, 121-125.

[83] Maitra, N. T.; Zhang, F.; Cave, R. J.; Burke, K. Double excitations within time-dependent density functional theory linear response. The Journal of Chemical Physics 2004, 120, 5932-5937.

[84] Elliott, P.; Goldson, S.; Canahui, C.; Maitra, N. T. Perspectives on double-excitations in TDDFT. Chemical Physics 2011, 391, 110-119.

[85] Filatov, M.; Huix-Rotllant, M.; Burghardt, I. Ensemble density functional theory method correctly describes bond dissociation, excited state electron transfer, and double excitations. The Journal of Chemical Physics 2015, 142, 184104.

[86] Sagredo, F.; Burke, K. Accurate double excitations from ensemble density functional calculations. The Journal of Chemical Physics 2018, 149, 134103. 
[87] Brandenburg, J. G.; Bannwarth, C.; Hansen, A.; Grimme, S. B97-3c: A revised low-cost variant of the B97-D density functional method. The Journal of Chemical Physics 2018, 148, 064104.

[88] Hirata, S.; Head-Gordon, M. Time-dependent density functional theory within the Tamm-Dancoff approximation. Chemical Physics Letters 1999, 314, 291-299.

[89] Weigend, F.; Ahlrichs, R. Balanced basis sets of split valence, triple zeta valence and quadruple zeta valence quality for $\mathrm{H}$ to $\mathrm{Rn}$ : Design and assessment of accuracy. Physical Chemistry Chemical Physics 2005, 7, $3297-3305$.

[90] Weigend, F. Hartree-Fock exchange fitting basis sets for H to Rn. Journal of Computational Chemistry 2008, 29, 167-175.

[91] Neese, F. Software update: the ORCA program system, version 4.0. Wiley Interdisciplinary Reviews: Computational Molecular Science 2018, 8, e1327.

[92] TURBOMOLE V7.0 2015, a development of University of Karlsruhe and Forschungszentrum Karlsruhe GmbH, 1989-2007, TURBOMOLE GmbH, since 2007; available from http://www.turbomole.com.

[93] Chantzis, A.; Laurent, A. D.; Adamo, C.; Jacquemin, D. Is the TammDancoff approximation reliable for the calculation of absorption and fluorescence band shapes? Journal of Chemical Theory and Computation 2013, 9, 4517-4525.

[94] Grimme, S. A simplified Tamm-Dancoff density functional approach for the electronic excitation spectra of very large molecules. The Journal of Chemical Physics 2013, 138, 244104.

[95] Dreuw, A.; Head-Gordon, M. Failure of time-dependent density functional theory for long-range charge-transfer excited states: 
the zincbacteriochlorin- bacteriochlorin and bacteriochlorophyllspheroidene complexes. Journal of the American Chemical Society 2004, 126, 4007-4016.

[96] Stein, T.; Kronik, L.; Baer, R. Reliable prediction of charge transfer excitations in molecular complexes using time-dependent density functional theory. Journal of the American Chemical Society 2009, 131, $2818-2820$.

[97] Meo, F. D.; Trouillas, P.; Adamo, C.; Sancho-García, J.-C. Application of recent double-hybrid density functionals to low-lying singlet-singlet excitation energies of large organic compounds. The Journal of Chemical Physics 2013, 139, 164104.

[98] Brémond, É.; Savarese, M.; Pérez-Jiménez, Á. J.; Sancho-García, J. C.; Adamo, C. Speed-up of the excited-state benchmarking: Double-hybrid density functionals as test cases. Journal of Chemical Theory and Computation 2017, 13, 5539-5551.

[99] Brémond, É.; Ottochian, A.; Pérez-Jiménez, Á. J.; Ciofini, I.; Scalmani, G.; Frisch, M. J.; Sancho-García, J. C.; Adamo, C. Assessing challenging intra-and inter-molecular charge-transfer excitations energies with double-hybrid density functionals. Journal of Computational Chemistry 2021, 42, 970-981.

[100] Kollmar, H.; Staemmler, V. Violation of Hund's rule by spin polarization in molecules. Theoretica Chimica Acta 1978, 48, 223-239. 
<smiles>C1=CC2=CC=CC3=CC=CC(=C1)N23</smiles>

$2 \mathrm{~T}-\mathrm{N}$<smiles>C1=CC2=NC=CC3=NC=CC(=N1)N23</smiles>

$2 \mathrm{~T}-4 \mathrm{~N}$<smiles>C1=NC2=NC=NC3=NC=NC(=N1)N23</smiles>

$2 \mathrm{~T}-7 \mathrm{~N}$

Figure 1: Chemical structures of the first set of studied molecules: 2T-N (or cyclazine), $2 \mathrm{~T}-4 \mathrm{~N}$, and $2 \mathrm{~T}-7 \mathrm{~N}$ (or heptazine). Hydrogen atoms are omitted for clarity. $2 \mathrm{~T}$ denotes the core and $\mathrm{xN}$ refers to the number of $\mathrm{C}$ sites substituted by $\mathrm{N}$ atoms.<smiles>C1=CC2=CC=NC3=NC=CC(=C1)N23</smiles>

$2 \mathrm{~T}-3 \mathrm{~N}$<smiles>C1=CC2=NC=NC3=NC=NC(=N1)N23</smiles>

$2 \mathrm{~T}-5 \mathrm{~N}$<smiles>C1=CC2=NC=NC3=NC=CC(=N1)N23</smiles>

$2 \mathrm{~T}-4_{\text {bis }} \mathrm{N}$<smiles>C1=CC2=CC=NC3=NC=NC(=N1)N23</smiles>

$2 \mathrm{~T}-5_{\text {bis }} \mathrm{N}$

Figure 2: Chemical structures of the second set of studied molecules: 2T-3N, $2 \mathrm{~T}-4_{\mathrm{bis}} \mathrm{N}, 2 \mathrm{~T}-5 \mathrm{~N}$, and $2 \mathrm{~T}-5_{\mathrm{bis}} \mathrm{N}$. Hydrogen atoms are omitted for clarity. $2 \mathrm{~T}$ denotes the core and $\mathrm{xN}$ refers to the number of $\mathrm{C}$ sites substituted by $\mathrm{N}$ atoms. 


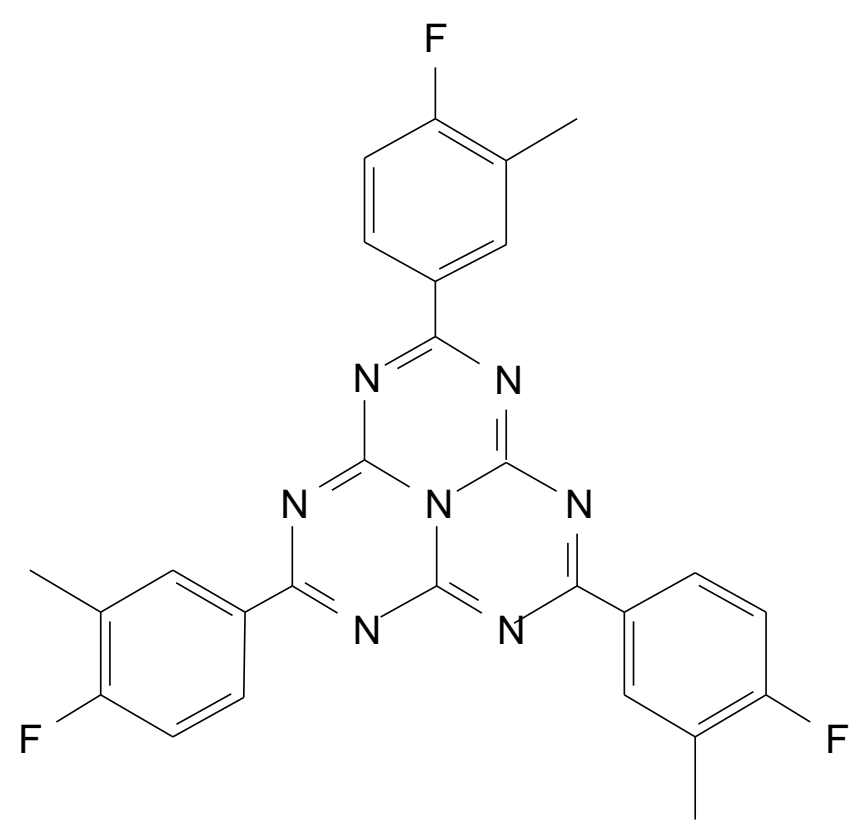

Figure 3: Chemical structure of the real-world HAP-3MF molecule. Hydrogen atoms are omitted for clarity. 
O†

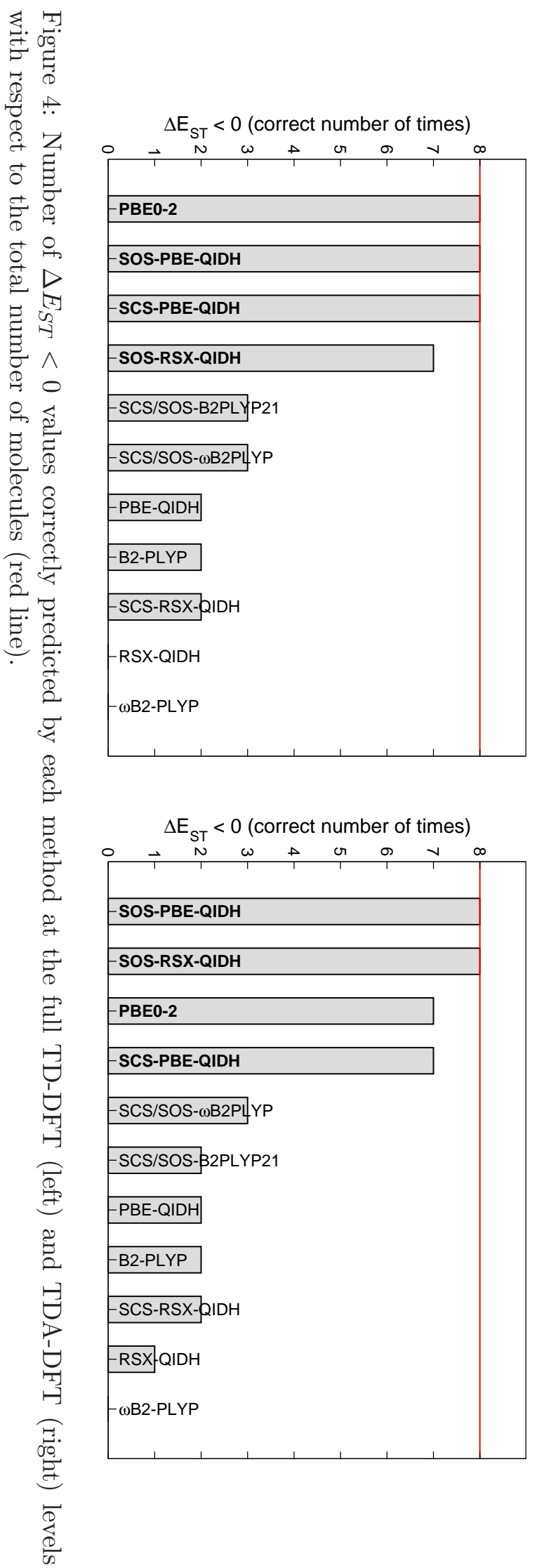



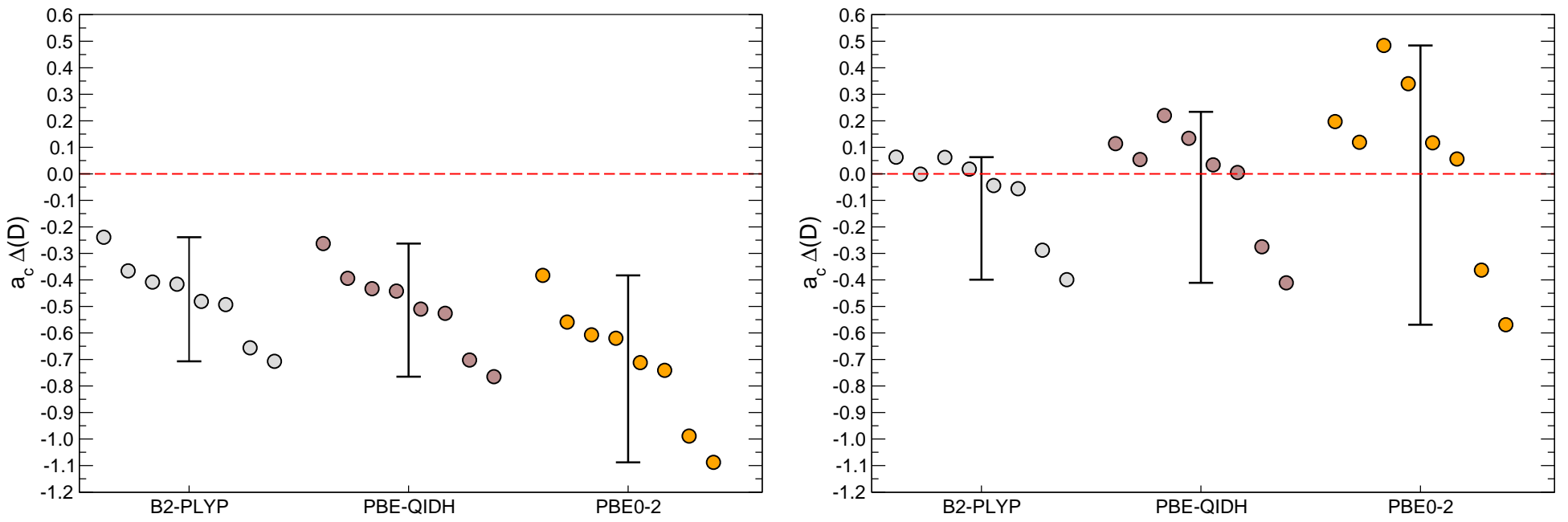

Figure 5: Magnitude (in eV) of the $a_{c} \Delta$ (D) term for the $S_{1} \leftarrow S_{0}$ (left) and $T_{1} \leftarrow S_{0}$ (right) as calculated by the standard functionals B2-PLYP $\left(a_{c}=0.27\right)$, PBE-QIDH $\left(a_{c}=1 / 3\right)$, and PBE0-2 $\left(a_{c}=1 / 2\right)$. For each of the functionals, the values for each molecule (circles) for $S_{1} \leftarrow S_{0}$ are ordered from low to high values, while the values for $T_{1} \leftarrow S_{0}$ correspond to that of the $S_{1} \leftarrow S_{0}$. The vertical bars denotes the maximum variation between the values for each functional and excitation. 
Table 1: Values of the coefficients weighting the different terms of the doublehybrid density functionals considered.

\begin{tabular}{|c|c|c|c|c|c|c|c|}
\hline Functional & $a_{x}$ & $a_{c}$ & $\omega$ & $c_{T}^{S S}$ & $c_{T}^{O S}$ & $c_{U}^{S S}$ & $c_{U}^{O S}$ \\
\hline B2-PLYP & 0.53 & 0.27 & - & - & - & - & - \\
\hline SCS/SOS-B2PLYP21 & 0.53 & 0.27 & - & 0.000 & 0.334 & 0.000 & 0.283 \\
\hline$\omega \mathrm{B} 2-\mathrm{PLYP}$ & 0.53 & 0.27 & 0.30 & - & - & - & - \\
\hline SCS/SOS- $\omega$ B2PLYP & 0.53 & 0.27 & 0.30 & 0.000 & 0.433 & 0.000 & 0.460 \\
\hline PBE-QIDH & $3^{-1 / 3}$ & $1 / 3$ & - & - & - & - & - \\
\hline SCS-PBE-QIDH & $3^{-1 / 3}$ & $1 / 3$ & - & 0.070 & 0.515 & 0.096 & 0.524 \\
\hline SOS-PBE-QIDH & $3^{-1 / 3}$ & $1 / 3$ & - & 0.000 & 0.547 & 0.000 & 0.573 \\
\hline RSX-QIDH & $3^{-1 / 3}$ & $1 / 3$ & 0.27 & - & - & - & - \\
\hline SCS-RSX-QIDH & $3^{-1 / 3}$ & $1 / 3$ & 0.27 & 0.174 & 0.541 & 0.586 & 0.424 \\
\hline SOS-RSX-QIDH & $3^{-1 / 3}$ & $1 / 3$ & 0.27 & 0.000 & 0.632 & 0.000 & 0.714 \\
\hline PBE0-2 & $2^{-1 / 3}$ & $1 / 2$ & - & - & - & - & - \\
\hline
\end{tabular}


Table 3: Vertical excitation energies (full TD-DFT) and associated $\Delta E_{S T}$ energy difference (all in eV) calculated with different methods, and with the def2-TZVP basis set unless otherwise noticed.

\begin{tabular}{|c|c|c|c|c|c|c|c|c|c|c|c|c|}
\hline \multirow[b]{2}{*}{ Method } & \multicolumn{3}{|c|}{$2 \mathrm{~T}-3 \mathrm{~N}$} & \multicolumn{3}{|c|}{$2 \mathrm{~T}-4_{\mathrm{bis}} \mathrm{N}$} & \multicolumn{3}{|c|}{$2 \mathrm{~T}-5 \mathrm{~N}$} & \multicolumn{3}{|c|}{$2 \mathrm{~T}-5_{\text {bis }} \mathrm{N}$} \\
\hline & $S_{1} \leftarrow S_{0}$ & $T_{1} \leftarrow S_{0}$ & $\Delta E_{S T}$ & $S_{1} \leftarrow S_{0}$ & $T_{1} \leftarrow S_{0}$ & $\Delta E_{S T}$ & $S_{1} \leftarrow S_{0}$ & $T_{1} \leftarrow S_{0}$ & $\Delta E_{S T}$ & $S_{1} \leftarrow S_{0}$ & $T_{1} \leftarrow S_{0}$ & $\Delta E_{S T}$ \\
\hline CIS & 2.617 & 2.102 & 0.52 & 3.056 & 2.405 & 0.65 & 3.442 & 2.772 & 0.67 & 3.427 & 2.759 & 0.67 \\
\hline CIS(D) & 1.592 & 1.921 & -0.33 & 1.946 & 2.305 & -0.36 & 2.193 & 2.663 & -0.47 & 2.122 & 2.549 & -0.43 \\
\hline SCS-CIS(D) & 1.356 & 1.798 & -0.44 & 1.709 & 2.172 & -0.46 & 1.948 & 2.520 & -0.57 & 1.882 & 2.420 & -0.54 \\
\hline SOS-CIS(D) & 0.918 & 1.516 & -0.60 & 1.264 & 1.891 & -0.64 & 1.489 & 2.230 & -0.74 & 1.423 & 2.136 & -0.71 \\
\hline B2-PLYP & 1.657 & 1.602 & 0.06 & 1.978 & 1.907 & 0.07 & 2.227 & 2.194 & 0.03 & 2.188 & 2.134 & 0.05 \\
\hline SCS/SOS-B2PLYP21 & 1.973 & 1.952 & 0.02 & 2.310 & 2.252 & 0.06 & 2.575 & 2.545 & 0.03 & 2.538 & 2.488 & 0.05 \\
\hline$\omega \mathrm{B} 2-\mathrm{PLYP}$ & 1.843 & 1.655 & 0.19 & 2.205 & 1.972 & 0.23 & 2.489 & 2.302 & 0.19 & 2.452 & 2.232 & 0.22 \\
\hline SCS/SOS- $\omega$ B2PLYP & 1.669 & 1.622 & 0.05 & 2.028 & 1.940 & 0.09 & 2.298 & 2.262 & 0.04 & 2.260 & 2.190 & 0.07 \\
\hline PBE-QIDH & 1.778 & 1.680 & 0.10 & 2.130 & 2.021 & 0.11 & 2.404 & 2.329 & 0.08 & 2.364 & 2.252 & 0.11 \\
\hline SCS-PBE-QIDH & 1.611 & 1.683 & -0.07 & 1.956 & 2.029 & -0.07 & 2.213 & 2.321 & -0.11 & 2.170 & 2.238 & -0.07 \\
\hline SOS-PBE-QIDH & 1.580 & 1.689 & -0.11 & 1.924 & 2.034 & -0.11 & 2.180 & 2.325 & -0.14 & 2.136 & 2.242 & -0.11 \\
\hline RSX-QIDH & 1.894 & 1.703 & 0.19 & 2.269 & 2.044 & 0.22 & 2.564 & 2.375 & 0.19 & 2.526 & 2.296 & 0.23 \\
\hline SCS-RSX-QIDH & 1.506 & 1.355 & 0.15 & 1.867 & 1.709 & 0.16 & 2.138 & 2.033 & 0.10 & 2.095 & 1.940 & 0.16 \\
\hline SOS-RSX-QIDH & 1.389 & 1.471 & -0.08 & 1.749 & 1.830 & -0.08 & 2.008 & 2.139 & -0.13 & 1.965 & 2.047 & -0.08 \\
\hline PBE0-2 & 1.706 & 1.758 & -0.05 & 2.059 & 2.192 & -0.13 & 2.326 & 2.433 & -0.11 & 2.278 & 2.331 & -0.05 \\
\hline EOM-CCSD/cc-pVDZ & 1.66 & 1.73 & -0.07 & 2.01 & 2.04 & -0.03 & 2.25 & 2.33 & -0.08 & 2.21 & 2.28 & -0.07 \\
\hline $\mathrm{SCS}-\mathrm{CC} 2$ & 1.690 & 1.891 & -0.20 & 2.050 & 2.225 & -0.17 & 2.308 & 2.541 & -0.23 & 2.258 & 2.477 & -0.22 \\
\hline
\end{tabular}

a Taken from Ref. 48 
Table 4: Vertical excitation energies (full TD-DFT) and associated $\Delta E_{S T}$ energy difference (all in $\mathrm{eV}$ ) calculated with different methods and basis sets.

\begin{tabular}{|c|c|c|c|c|c|c|}
\hline \multirow[b]{3}{*}{ Method } & \multicolumn{6}{|c|}{ HAP-3MF } \\
\hline & \multicolumn{3}{|c|}{ def2-SVP } & \multicolumn{3}{|c|}{ def2-TZVP } \\
\hline & $S_{1} \leftarrow S_{0}$ & $T_{1} \leftarrow S_{0}$ & $\Delta E_{S T}$ & $S_{1} \leftarrow S_{0}$ & $T_{1} \leftarrow S_{0}$ & $\Delta E_{S T}$ \\
\hline CIS & 4.377 & 3.243 & 1.13 & 4.446 & 3.227 & 1.22 \\
\hline CIS(D) & 2.487 & 3.040 & -0.55 & 2.557 & 3.092 & -0.54 \\
\hline SCS-CIS(D) & 2.248 & 2.966 & -0.72 & 2.325 & 3.014 & -0.69 \\
\hline SOS-CIS(D) & 1.799 & 2.695 & -0.90 & 1.839 & 2.716 & -0.88 \\
\hline B2-PLYP & 2.657 & 2.730 & -0.07 & 2.705 & 2.753 & -0.05 \\
\hline SCS/SOS-B2PLYP21 & 3.042 & 3.143 & -0.10 & 3.144 & 3.209 & -0.06 \\
\hline$\omega B 2-P L Y P$ & 3.073 & 3.077 & -0.00 & 3.153 & 3.122 & 0.03 \\
\hline SCS/SOS- $\omega$ B2PLYP & 2.847 & 3.023 & -0.18 & 2.936 & 3.070 & -0.13 \\
\hline PBE-QIDH & 2.907 & 2.978 & -0.07 & 2.961 & 2.997 & -0.04 \\
\hline SCS-PBE-QIDH & 2.666 & 2.921 & -0.26 & 2.731 & 2.946 & -0.22 \\
\hline SOS-PBE-QIDH & 2.631 & 2.932 & -0.30 & 2.701 & 2.958 & -0.26 \\
\hline RSX-QIDH & 3.173 & 3.186 & -0.01 & 3.232 & 3.213 & 0.02 \\
\hline SCS-RSX-QIDH & 2.627 & 2.726 & -0.10 & 2.667 & 2.725 & -0.06 \\
\hline SOS-RSX-QIDH & 2.508 & 2.869 & -0.36 & 2.574 & 2.880 & -0.31 \\
\hline PBE0-2 & 2.783 & 2.991 & -0.21 & 2.841 & 3.014 & -0.17 \\
\hline $\operatorname{ADC}(2) / c c-p V D Z^{a}$ & 2.58 & 2.82 & -0.24 & & & \\
\hline SCS-CC2/cc-pVDZ & 2.82 & 3.18 & -0.36 & & & \\
\hline SCS-CC2 & 2.773 & 3.170 & -0.40 & 3.082 & 3.237 & -0.16 \\
\hline
\end{tabular}

a Taken from Ref. 52 
Table 5: Vertical excitation energies (under the Tamm-Dancoff approximation or TDA-DFT) and associated $\Delta E_{S T}$ energy difference (all in $\mathrm{eV}$ ) calculated with different methods, and with the def2-TZVP basis set unles otherwise noticed.

\begin{tabular}{|c|c|c|c|c|c|c|c|c|c|}
\hline \multirow[b]{2}{*}{ Method } & \multicolumn{3}{|c|}{$2 \mathrm{~T}-\mathrm{N}$} & \multicolumn{3}{|c|}{$2 \mathrm{~T}-4 \mathrm{~N}$} & \multicolumn{3}{|c|}{$2 \mathrm{~T}-7 \mathrm{~N}$} \\
\hline & $S_{1} \leftarrow S_{0}$ & $T_{1} \leftarrow S_{0}$ & $\Delta E_{S T}$ & $S_{1} \leftarrow S_{0}$ & $T_{1} \leftarrow S_{0}$ & $\Delta E_{S T}$ & $S_{1} \leftarrow S_{0}$ & $T_{1} \leftarrow S_{0}$ & $\Delta E_{S T}$ \\
\hline B2-PLYP & 1.155 & 1.132 & 0.02 & 2.139 & 2.028 & 0.11 & 2.768 & 2.813 & -0.04 \\
\hline SCS/SOS-B2PLYP21 & 1.444 & 1.416 & 0.03 & 2.493 & 2.343 & 0.15 & 3.169 & 3.202 & -0.03 \\
\hline$\omega \mathrm{B} 2-\mathrm{PLYP}$ & 1.310 & 1.219 & 0.09 & 2.449 & 2.213 & 0.24 & 3.170 & 3.124 & 0.05 \\
\hline SCS/SOS- $\omega$ B2PLYP & 1.176 & 1.193 & -0.02 & 2.313 & 2.197 & 0.12 & 2.974 & 3.087 & -0.11 \\
\hline PBE-QIDH & 1.241 & 1.203 & 0.04 & 2.327 & 2.173 & 0.15 & 3.031 & 3.065 & -0.03 \\
\hline SCS-PBE-QIDH & 1.112 & 1.198 & -0.09 & 2.186 & 2.173 & 0.01 & 2.817 & 3.030 & -0.21 \\
\hline SOS-PBE-QIDH & 1.089 & 1.204 & -0.12 & 2.167 & 2.183 & -0.02 & 2.786 & 3.041 & -0.26 \\
\hline RSX-QIDH & 1.345 & 1.263 & 0.08 & 2.520 & 2.283 & 0.24 & 3.278 & 3.252 & 0.03 \\
\hline SCS-RSX-QIDH & 1.007 & 0.997 & 0.01 & 2.126 & 2.013 & 0.11 & 2.793 & 2.872 & -0.08 \\
\hline SOS-RSX-QIDH & 0.943 & 1.084 & -0.14 & 2.076 & 2.114 & -0.04 & 2.687 & 2.990 & -0.30 \\
\hline PBE0-2 & 1.194 & 1.241 & -0.05 & 2.281 & 2.237 & 0.04 & 2.863 & 3.029 & -0.17 \\
\hline
\end{tabular}


Table 6: Vertical excitation energies (under the Tamm-Dancoff approximation or TDA-DFT) and associated $\Delta E_{S T}$ energy difference (all in eV) calculated with different methods, and with the def2-TZVP basis set unless otherwise noticed.

\begin{tabular}{|c|c|c|c|c|c|c|c|c|c|c|c|c|}
\hline \multirow[b]{2}{*}{ Method } & \multicolumn{3}{|c|}{$2 \mathrm{~T}-3 \mathrm{~N}$} & \multicolumn{3}{|c|}{$2 \mathrm{~T}-4_{\mathrm{bis}} \mathrm{N}$} & \multicolumn{3}{|c|}{$2 \mathrm{~T}-5 \mathrm{~N}$} & \multicolumn{3}{|c|}{$2 \mathrm{~T}-5_{\text {bis }} \mathrm{N}$} \\
\hline & $S_{1} \leftarrow S_{0}$ & $T_{1} \leftarrow S_{0}$ & $\Delta E_{S T}$ & $S_{1} \leftarrow S_{0}$ & $T_{1} \leftarrow S_{0}$ & $\Delta E_{S T}$ & $S_{1} \leftarrow S_{0}$ & $T_{1} \leftarrow S_{0}$ & $\Delta E_{S T}$ & $S_{1} \leftarrow S_{0}$ & $T_{1} \leftarrow S_{0}$ & $\Delta E_{S T}$ \\
\hline B2-PLYP & 1.693 & 1.640 & 0.05 & 2.017 & 1.943 & 0.07 & 2.263 & 2.231 & 0.03 & 2.224 & 2.179 & 0.04 \\
\hline SCS/SOS-B2PLYP21 & 2.021 & 1.942 & 0.08 & 2.362 & 2.248 & 0.11 & 2.624 & 2.548 & 0.08 & 2.586 & 2.496 & 0.09 \\
\hline$\omega \mathrm{B} 2-\mathrm{PLYP}$ & 1.933 & 1.782 & 0.15 & 2.296 & 2.103 & 0.19 & 2.579 & 2.421 & 0.16 & 2.540 & 2.366 & 0.17 \\
\hline SCS/SOS- $\omega$ B2PLYP & 1.784 & 1.753 & 0.03 & 2.143 & 2.074 & 0.07 & 2.413 & 2.388 & 0.02 & 2.372 & 2.332 & 0.04 \\
\hline PBE-QIDH & 1.838 & 1.757 & 0.08 & 2.192 & 2.082 & 0.11 & 2.466 & 2.403 & 0.06 & 2.425 & 2.343 & 0.08 \\
\hline SCS-PBE-QIDH & 1.687 & 1.745 & -0.06 & 2.033 & 2.070 & -0.04 & 2.292 & 2.385 & -0.09 & 2.247 & 2.322 & -0.08 \\
\hline SOS-PBE-QIDH & 1.662 & 1.751 & -0.09 & 2.009 & 2.076 & -0.07 & 2.265 & 2.391 & -0.13 & 2.219 & 2.328 & -0.11 \\
\hline RSX-QIDH & 1.992 & 1.847 & 0.14 & 2.367 & 2.177 & 0.19 & 2.662 & 2.512 & 0.15 & 2.622 & 2.452 & 0.17 \\
\hline SCS-RSX-QIDH & 1.609 & 1.563 & 0.05 & 1.969 & 1.897 & 0.07 & 2.240 & 2.221 & 0.02 & 2.196 & 2.155 & 0.04 \\
\hline SOS-RSX-QIDH & 1.538 & 1.654 & -0.12 & 1.895 & 1.987 & -0.09 & 2.156 & 2.311 & -0.16 & 2.109 & 2.245 & -0.14 \\
\hline PBE0-2 & 1.781 & 1.808 & -0.03 & 2.136 & 2.147 & -0.01 & 2.403 & 2.479 & -0.08 & 2.355 & 2.406 & -0.05 \\
\hline
\end{tabular}


Table 7: Vertical excitation energies (under the Tamm-Dancoff approximation or TDADFT) and associated $\Delta E_{S T}$ energy difference (all in eV) calculated with different methods and basis sets.

\begin{tabular}{|c|c|c|c|c|c|c|}
\hline \multirow[b]{3}{*}{ Method } & \multicolumn{6}{|c|}{ HAP-3MF } \\
\hline & \multicolumn{3}{|c|}{ def2-SVP } & \multicolumn{3}{|c|}{ def2-TZVP } \\
\hline & $S_{1} \leftarrow S_{0}$ & $T_{1} \leftarrow S_{0}$ & $\Delta E_{S T}$ & $S_{1} \leftarrow S_{0}$ & $T_{1} \leftarrow S_{0}$ & $\Delta E_{S T}$ \\
\hline B2-PLYP & 2.684 & 2.764 & -0.08 & 2.730 & 2.793 & -0.06 \\
\hline SCS/SOS-B2PLYP21 & 3.084 & 3.159 & -0.08 & 3.175 & 3.227 & -0.05 \\
\hline$\omega \mathrm{B} 2-\mathrm{PLYP}$ & 3.143 & 3.278 & -0.14 & 3.220 & 3.209 & 0.01 \\
\hline SCS/SOS- $\omega$ B2PLYP & 2.941 & 3.235 & -0.29 & 3.027 & 3.161 & -0.13 \\
\hline PBE-QIDH & 2.956 & 3.042 & -0.09 & 3.008 & 3.065 & -0.06 \\
\hline SCS-PBE-QIDH & 2.732 & 2.984 & -0.25 & 2.795 & 3.012 & -0.22 \\
\hline SOS-PBE-QIDH & 2.702 & 2.995 & -0.29 & 2.771 & 3.025 & -0.25 \\
\hline RSX-QIDH & 3.240 & 3.275 & -0.04 & 3.307 & 3.312 & -0.00 \\
\hline SCS-RSX-QIDH & 2.719 & 2.844 & -0.12 & 2.757 & 2.855 & -0.10 \\
\hline SOS-RSX-QIDH & 2.636 & 2.978 & -0.34 & 2.699 & 3.002 & -0.30 \\
\hline PBE0-2 & 2.853 & 3.059 & -0.21 & 2.908 & 3.085 & -0.18 \\
\hline
\end{tabular}


Table 8: MSD, MAD, and RMSD values (in eV) for the double-hybrid density functionals selected, calculated with the with the def2-TZVP basis set and with respect to the SCS-CC2 values taken as reference.

\begin{tabular}{|c|c|c|c|c|c|c|c|c|c|}
\hline \multirow[b]{2}{*}{ Method } & \multicolumn{3}{|c|}{$S_{1} \leftarrow S_{0}$} & \multicolumn{3}{|c|}{$T_{1} \leftarrow S_{0}$} & \multicolumn{3}{|c|}{$\Delta E_{S T}$} \\
\hline & MSD & MAD & RMSD & MSD & MAD & RMSD & MSD & MAD & RMSD \\
\hline B2-PLYP & -0.11 & 0.11 & 0.16 & -0.35 & 0.35 & 0.36 & 0.24 & 0.24 & 0.25 \\
\hline SCS/SOS-B2PLYP21 & 0.24 & 0.24 & 0.25 & 0.02 & 0.04 & 0.05 & 0.22 & 0.22 & 0.23 \\
\hline$\omega \mathrm{B} 2-\mathrm{PLYP}$ & 0.15 & 0.15 & 0.16 & -0.23 & 0.23 & 0.23 & 0.38 & 0.38 & 0.39 \\
\hline SCS/SOS- $\omega$ B2PLYP & -0.03 & 0.04 & 0.06 & -0.26 & 0.26 & 0.27 & 0.23 & 0.23 & 0.24 \\
\hline PBE-QIDH & 0.06 & 0.09 & 0.09 & -0.22 & 0.22 & 0.22 & 0.28 & 0.28 & 0.29 \\
\hline SCS-PBE-QIDH & -0.13 & 0.13 & 0.15 & -0.22 & 0.22 & 0.23 & 0.10 & 0.10 & 0.12 \\
\hline SOS-PBE-QIDH & -0.16 & 0.16 & 0.18 & -0.22 & 0.22 & 0.22 & 0.06 & 0.06 & 0.09 \\
\hline RSX-QIDH & 0.22 & 0.22 & 0.23 & -0.16 & 0.16 & 0.17 & 0.38 & 0.38 & 0.39 \\
\hline SCS-RSX-QIDH & -0.21 & 0.21 & 0.23 & -0.53 & 0.53 & 0.53 & 0.32 & 0.32 & 0.33 \\
\hline SOS-RSX-QIDH & -0.33 & 0.33 & 0.34 & -0.40 & 0.40 & 0.40 & 0.07 & 0.07 & 0.11 \\
\hline PBE0-2 & -0.02 & 0.05 & 0.09 & -0.13 & 0.13 & 0.14 & 0.11 & 0.11 & 0.13 \\
\hline
\end{tabular}


Table 9: Magnitude (in $\mathrm{eV}$ ) of the $a_{c} \Delta(\mathrm{D})$ term, as calculated by various (standard) double-hybrid functionals at the full TD-DFT level.

\begin{tabular}{lcccccccc}
\hline & \multicolumn{2}{c}{ B2-PLYP } & & \multicolumn{2}{c}{ PBE-QIDH } & & \multicolumn{2}{c}{ PBE0-2 } \\
\cline { 2 - 3 } Molecule & $S_{1} \leftarrow S_{0}$ & $T_{1} \leftarrow S_{0}$ & & $S_{1} \leftarrow S_{0}$ & $T_{1} \leftarrow S_{0}$ & & $S_{1} \leftarrow S_{0}$ & $T_{1} \leftarrow S_{0}$ \\
\hline $2 \mathrm{~T}-\mathrm{N}$ & -0.239 & 0.063 & & -0.263 & 0.114 & & -0.383 & 0.197 \\
$2 \mathrm{~T}-4 \mathrm{~N}$ & -0.408 & 0.062 & & -0.433 & 0.220 & & -0.607 & 0.484 \\
$2 \mathrm{~T}-7 \mathrm{~N}$ & -0.656 & -0.288 & & -0.702 & -0.275 & & -0.989 & -0.363 \\
$2 \mathrm{~T}-3 \mathrm{~N}$ & -0.366 & -0.001 & & -0.394 & 0.054 & & -0.559 & 0.119 \\
$2 \mathrm{~T}-4_{\text {bis }} \mathrm{N}$ & -0.416 & 0.018 & & -0.422 & 0.134 & & -0.620 & 0.340 \\
$2 \mathrm{~T}-5 \mathrm{~N}$ & -0.481 & -0.044 & & -0.510 & 0.034 & & -0.712 & 0.117 \\
$2 \mathrm{~T}-5_{\text {bis }} \mathrm{N}$ & -0.493 & -0.056 & & -0.526 & 0.005 & & -0.741 & 0.056 \\
HAP-3MF & -0.707 & -0.399 & & -0.765 & -0.411 & & -1.088 & -0.569 \\
\hline
\end{tabular}


Table 10: Magnitude (in eV) of the $a_{c} \Delta(\mathrm{D})$ term, as calculated by various (spin-scaled) double-hybrid functionals at the full TD-DFT level.

\begin{tabular}{lrrrrr}
\hline & \multicolumn{2}{c}{ SCS-PBE-QIDH } & & \multicolumn{2}{c}{ SOS-PBE-QIDH } \\
\cline { 2 - 3 } \cline { 6 - 6 } Molecule & $S_{1} \leftarrow S_{0}$ & $T_{1} \leftarrow S_{0}$ & & $S_{1} \leftarrow S_{0}$ & $T_{1} \leftarrow S_{0}$ \\
\hline $2 \mathrm{~T}-\mathrm{N}$ & -0.409 & 0.131 & & -0.440 & 0.139 \\
$2 \mathrm{~T}-4 \mathrm{~N}$ & -0.585 & 0.264 & & -0.610 & 0.275 \\
$2 \mathrm{~T}-7 \mathrm{~N}$ & -0.935 & -0.303 & & -0.972 & -0.292 \\
$2 \mathrm{~T}-3 \mathrm{~N}$ & -0.560 & 0.056 & & -0.591 & 0.063 \\
$2 \mathrm{~T}-4_{\text {bis }} \mathrm{N}$ & -0.616 & 0.143 & & -0.647 & 0.148 \\
$2 \mathrm{~T}-5 \mathrm{~N}$ & -0.701 & 0.026 & & -0.734 & 0.030 \\
$2 \mathrm{~T}-5_{\text {bis }} \mathrm{N}$ & -0.720 & -0.009 & & -0.754 & -0.005 \\
HAP-3MF & -1.006 & -0.468 & & -1.041 & -0.457 \\
\hline
\end{tabular}




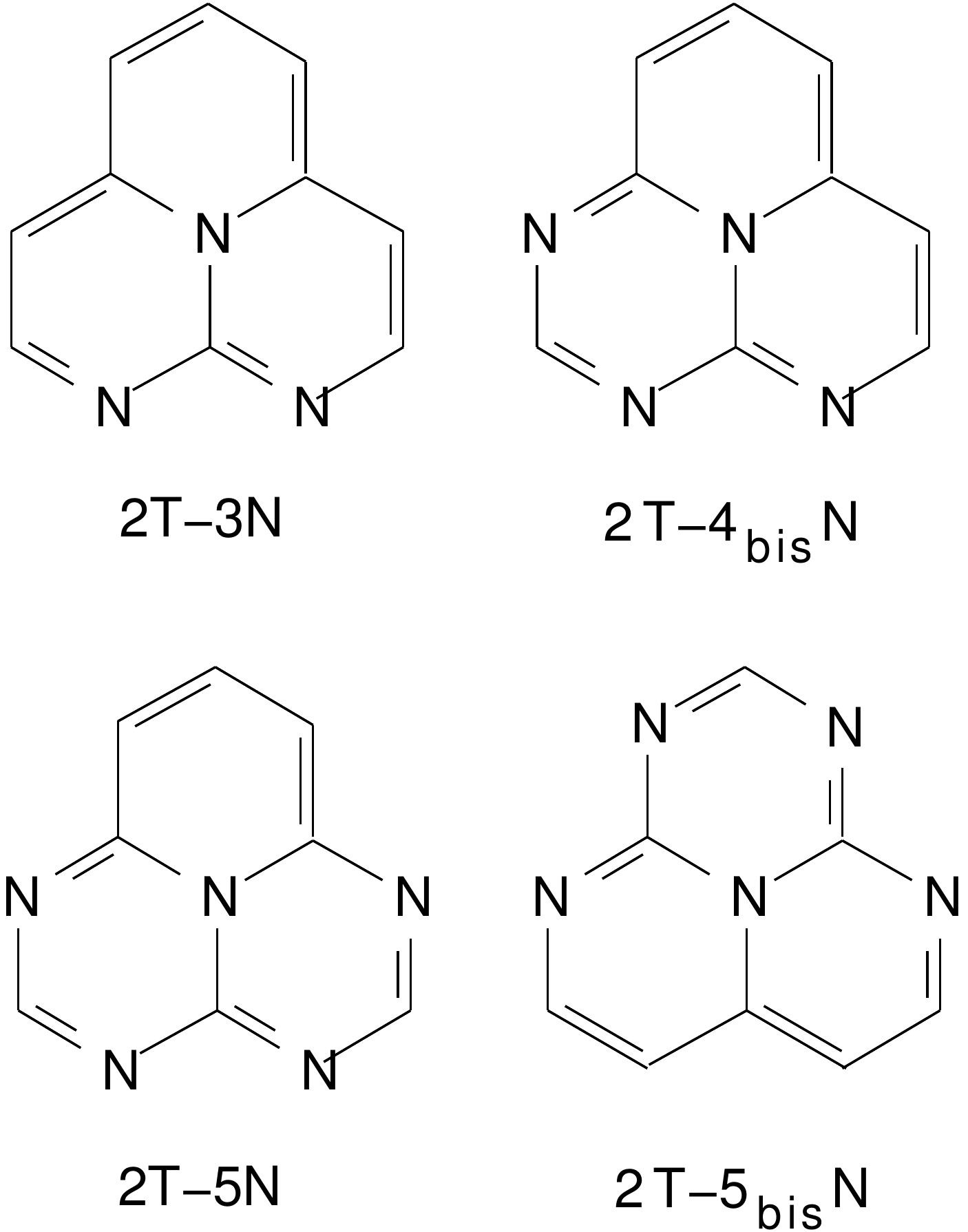



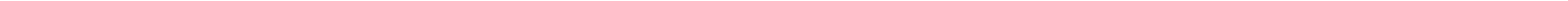




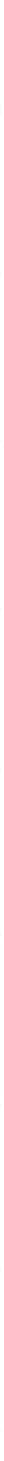


